\title{
1 Perception is associated with the brain's metabolic \\ 2 response to sensory stimulation
}

3 Mauro DiNuzzo ${ }^{1, \S}$, Silvia Mangia ${ }^{2, \S}$, Marta Moraschi $^{3}$, Daniele Mascali, ${ }^{1,4}$, Gisela E. Hagberg ${ }^{5}$,

4 Federico Giove ${ }^{1,6,{ }^{*}}$

$6 \quad{ }^{1}$ Museo Storico della Fisica e Centro Studi e Ricerche Enrico Fermi, 00184 Rome, Italy

$7 \quad{ }^{2}$ Center for Magnetic Resonance Research, Department of Radiology, University of Minnesota,

8 Minneapolis, MN 55455, USA

$9 \quad{ }^{3}$ Department of radiation oncology, Campus Bio-Medico University of Rome, 00128 Rome, Italy

$10{ }^{4}$ Dipartimento di Neuroscienze, Imaging e Scienze Cliniche, Università Gabriele D’Annunzio,

1166100 Chieti, Italy

$12{ }^{5}$ High-Field Magnetic Resonance, Max Planck Institute for Biological Cybernetics and

13 Biomedical Magnetic Resonance, University Hospital Tübingen, 72076 Tübingen, Germany

$14{ }^{6}$ Fondazione Santa Lucia IRCCS, 00179 Rome, Italy

$15 \S$ These authors contributed equally to this work.

$17{ }^{*}$ correspondence to:

18 Federico Giove, Ph.D.

19 Magnetic Resonance for Brain Investigation Laboratory

20 c/o Fondazione Santa Lucia 354, 00179 Rome, Italy

21 Phone: +39 0651501157

22 


\section{Competing interests}

24 The authors declare no competing financial interests.

25

26 Keywords: central visual system • visual perception • isoluminant chromatic flickering • human

27 brain metabolism $\cdot$ lactate $\cdot$ BOLD fMRI $\cdot$ single voxel ${ }^{1} \mathrm{H}$-fMRS

28

29 Running title: Cortical metabolic response during visual perception

30

$31 \quad$ Abstract word count: 187

32 Word count: about 6900 (Introduction, Results, Discussion, Methods).

33 Number of Tables: 0

34 Number of figures: 3

35 References count: 118

36 Supplementary Information: 13 Figure Supplement, 6 Source Data, 1 Video

37

38

Article type: Research Article

39

40

41

42

43 Authors Contact and ORCiD Information 
45 Mauro DiNuzzo

46 ORCiD: https://orcid.org/0000-0003-0181-5597

47 email: mauro.dinuzzo@neuroenergetics.org

48 Silvia Mangia

49 ORCiD: https://orcid.org/0000-0001-6341-4516

50 email: mangia@umn.edu

51 Marta Moraschi

52 ORCiD: https://orcid.org/0000-0003-4789-6636

53 email: martamoras@gmail.com

54 Daniele Mascali

55 ORCiD: https://orcid.org/0000-0003-1269-6060

56 email: daniele.mascali@unich.it

57

58 Gisela E. Hagberg

59 ORCiD: https://orcid.org/0000-0003-2176-7086

60 email: gisela.hagberg@tuebingen.mpg.de

61 Federico Giove

62 ORCiD: https://orcid.org/0000-0002-6934-3146

63 email: federico.giove@cref.it

64

\section{Highlights (if required by the Journal):}

66 - Processing of visual stimulation upregulates energy metabolism in V1

67 - Temporal frequency of flickering modulates cortical activation and perception

68 - Perceived and unperceived flickering results in distinct metabolic responses in V1

69 - Lactate is proposed as an indicator of sensory perception 


\section{Abstract}

72 Processing of incoming sensory stimulation triggers an increase of cerebral perfusion and blood

73 oxygenation (neurovascular response) as well as an alteration of the metabolic neurochemical

74 profile (neurometabolic response). Here we show in human primary visual cortex (V1) that

75 perceived and unperceived isoluminant chromatic flickering stimuli designed to have similar

76 neurovascular responses as measured by blood oxygenation level dependent functional MRI

77 (BOLD-fMRI) have markedly different neurometabolic responses as measured by functional

78 MRS. In particular, a significant regional buildup of lactate, an index of aerobic glycolysis, and

79 glutamate, an index of malate-aspartate shuttle, occurred in V1 only when the flickering was

80 perceived, without any relation with behavioral or physiological variables. Whereas the BOLD-

81 fMRI signal in $\mathrm{V} 1$, a proxy for input to $\mathrm{V} 1$, was insensitive to flickering perception by design, the

82 BOLD-fMRI signal in secondary visual areas was larger during perceived than unperceived

83 flickering, indicating increased output from $\mathrm{V} 1$. These results demonstrate that the upregulation

84 of energy metabolism induced by visual stimulation depends on the type of information

85 processing taking place in $\mathrm{V} 1$, and that $1 \mathrm{H}$-fMRS provides unique information about local

86 input/output balance that is not measured by BOLD fMRI.

\section{Significance statement (if required by the Journal)}

89 Visual perception has a measurable metabolic effect in the primary visual cortex (V1). Here we

90 show that the upregulation of energy metabolism induced by isoluminant chromatic flickering is

91 associated with subjective visual perception. Within V1, perceived and unperceived stimuli that

92 are contrast-matched to produce similar blood-oxygenation level-dependent (BOLD) fMRI

93 responses elicit clearly distinct changes in the neurochemical profile. Specifically, regional 
94 accumulations of lactate (an index of aerobic glycolysis) and glutamate (an index of malate95 aspartate shuttle) develop in V1 only during perceived stimuli. Our results imply a 96 dissociation between neurometabolic and neurovascular response, and indicate that the

97 upregulation of energy metabolism induced by visual stimulation depends on the type of 98 information processing taking place in $\mathrm{V} 1$.

99

100 Impact statement (40 words)

101 Perceived and unperceived visual stimuli that are contrast-matched to produce similar BOLD 102 responses in V1 elicit clearly distinct changes in the neurochemical profile, implying a 103 perception-related dissociation between neurometabolic and neurovascular response. 


\section{Introduction}

107 In the brain, sensory stimulation is associated with a substantial increase of regional functional

108 hyperaemia (i.e. cerebral blood flow, CBF) as well as energy metabolism of glucose, the main 109 cerebral energy substrate (Siesjo 1978). The metabolic response to stimulation includes an

110 oxidative component, as measured by the cerebral metabolic rate of oxygen $\left(\mathrm{CMRO}_{2}\right)$, and a

111 non-oxidative component, as reflected by lactate accumulation (Mangia et al. 2009). Cortical

112 lactate levels have been shown to increase during visual stimulation, simultaneously to the

113 acceleration of the malate-aspartate shuttle, a process termed aerobic glycolysis (i.e. lactate

114 production independent of oxygen availability) (Bednarik et al. 2015; Bednarik et al. 2018; Boillat

115 et al. 2020; Fernandes et al. 2020; Lin et al. 2010; Lin et al. 2012; Mangia et al. 2007a; Schaller

116 et al. 2013; Schaller et al. 2014). Despite intense research, the neurophysiological mechanisms

117 underlying the up-regulation of glycolytic metabolism of glucose are still largely unknown (Dienel

118 2019a). Most importantly, the impact of information processing to the metabolic response of the

119 cerebral cortex to sensory stimulation has not been fully investigated thus far. In particular, 120 nothing is known about the modulatory effect exerted by the perception of different stimuli on 121 regional brain energy metabolism.

122 Sensory perception is thought to rely on the complex interplay of neural circuits that process 123 information in a cortical layer- and area-mediated manner involving thalamo-cortical, 124 intracortical, cortico-cortical and cortico-thalamic feedforward/feedback loops (D'Souza and 125 Burkhalter 2017). Sensory stimuli transduced by sensory organs reach specific thalamic nuclei 126 that relay information to primary sensory cortices, which in turn filter and eventually transmit 127 information to secondary sensory areas (Crick and Koch 1995). These transactions are 128 dependent on the particular features of different incoming stimuli, thus it is possible that the 
relevant neurovascular and neurometabolic counterparts are correspondingly distinct (Lauritzen

130 2001).

131 The thalamic lateral geniculate nucleus (LGN) mediates visual stimuli with temporal frequencies

132 at least up to $90 \mathrm{~Hz}$ to the layer IV of V1 (Gur and Snodderly 1997; Herrmann 2001; Norcia et

133 al. 2015; Pastor et al. 2003; Regan 1989; Vialatte et al. 2010), which in turn relays to output

134 layers II/III and V where temporal filtering occurs (Sachidhanandam et al. 2013), consistent with

135 the notion that visual perception requires the activation of visual areas downstream V1 (i.e.

136 secondary visual areas). In agreement with these arguments, it has been repeatedly reported

137 that invisible visual flickering is still able to activate V1 even without any perceptual effects (Alais

138 et al. 2016), as revealed by in vivo electrophysiology in non-human primates (Gur and

139 Snodderly 1997) as well as behavioral evidence (Lorenceau 1987) and blood-oxygenation level

140 dependent (BOLD) functional magnetic resonance imaging (fMRI) (Jiang et al. 2007) in

141 humans. High $(30 \mathrm{~Hz})$ frequency visual stimulation has been found to selectively suppress multi-

142 unit activity (MUA) in cat $\mathrm{V} 1$ as compared to low frequency $(4 \mathrm{~Hz})$ visual stimulation

143 (Viswanathan and Freeman 2007). Importantly, local field potentials (LFPs) and tissue oxygen

144 response, which directly contribute to the generation of the BOLD signals (Logothetis et al.

145 2001), were preserved at both frequencies.

146 In the present study, we combined BOLD-fMRI and proton functional magnetic resonance 147 spectroscopy (1H-fMRS) in humans and exploited the well known effect of temporal frequency 148 on visual perception. Specifically, we examined the functional and metabolic responses of the 149 primary visual cortex (V1) to perceived or unperceived isoluminant chromatic flickering 150 stimulation obtained by using temporal frequency either below (7.5 Hz; PF, perceived flickering) 151 or above (30 Hz; UF, unperceived flickering) the critical flicker fusion (CFF) threshold of $\sim 15 \mathrm{~Hz}$ 152 for rod-mediated vision (Hecht and Shlaer 1936). Based on experimental evidence and 
153 metabolic modeling, we have previously proposed that the local input-output balance between 154 neuronal synaptic/spiking (or subthreshold/suprathreshold) activity is a primary determinant in 155 the up-regulation of aerobic glycolysis (DiNuzzo and Giove 2012; DiNuzzo et al. 2011; DiNuzzo 156 and Nedergaard 2017). We thus hypothesized that the loss of visual perception is accompanied 157 by fundamental changes in the metabolic responses of human $\mathrm{V} 1$. 


\section{Results}

\section{Subjects perception of the visual stimuli}

161 To achieve perceptual isoluminance between green and red color (necessary for the UF 162 condition), we adjusted the brightness of the green color for each individual subject, which was 163 remarkably similar across subjects (green/red brightness ratio $71.9 \pm 1.2 \%$, range $70.1 \%$ to $16473.5 \%$ ) (Figure 1-Source Data 1). After this procedure, 100\% of the subjects confirmed that 165 their perception of the $30 \mathrm{~Hz}$ frequency stimulus steadiness was equivalent to the resting 166 condition. Overall, the subject's perception was a gray/colored checkerboard that in the colored 167 squares showed either a fast green and red alternation during PF epochs, or a static yellow 168 during UF epochs (Video 1). As a further confirmation, while in the scanner the subjects were 169 unable to distinguish the $30 \mathrm{~Hz}$ red-green flickering checkerboard (used in the actual 170 experiments) from a color-matched static yellow checkerboard (used for testing only).

171 Specifically, the perception of the steady yellow color versus the $30 \mathrm{~Hz}$ red-green flickering was 172 indistinguishable, as assessed by asking the subjects to guess the origin of the stimulus for 10 173 consecutive trials (average of correct responses $52 \pm 16 \%$, not different from chance level, $174 \mathrm{p}=0.62$ ). All subjects reported to distinctly perceive the green and red color when the 175 checkerboard was flickering at $7.5 \mathrm{~Hz}$. None of the subjects perceived the intrinsic flickering of 176 the screen due to the refresh rate $(60 \mathrm{~Hz})$.

\section{Subjects attention and task performance}

178 To examine the possible modulation of the BOLD signal by attention (Watanabe et al. 2011), we 179 measured pupillometry and task-performance data during all experiments. All subjects 180 maintained gaze on the fixation cross within 0.2-0.3 degrees during all fMRI (Figure 1-Figure 181 Supplement 1) and 1H-fMRS (Figure 3-Figure Supplement 1) epochs, with no difference in 182 average gaze location between the different stimulations (One-way ANOVA, $p=0.96, p=0.38$, for 
$183 \mathrm{fMRI}$ run 1 and 2, respectively, and $\mathrm{p}=0.29, \mathrm{p}=0.19$ for $1 \mathrm{H}$-fMRS run 1 and 2 , respectively). 184 Pupil diameter, an index of the noradrenergic tone (DiNuzzo et al. 2019), was fairly stable at 185 around 6.5-7 $\mathrm{mm}$ on average during all fMRI (Figure 1-Figure Supplement 2) and 1H-fMRS 186 (Figure 3-Figure Supplement 2) epochs (One-way ANOVA, $p=0.99, p=0.98$, for fMRI run 1 187 and 2, respectively, and $\mathrm{p}=0.98, \mathrm{p}=0.96$ for $1 \mathrm{H}$-fMRS run 1 and 2 , respectively) across 188 conditions (see also Video 1), indicating that the modulation of perception by noradrenaline 189 (Gelbard-Sagiv et al. 2018) was minimal in our experimental conditions. The hit/miss ratio for 190 the cross rotation task during the stimulation protocol was essentially 1.0 , as expected due to 191 the simplicity of the task, for the entire duration of the experiment. In particular, the delay of the 192 response was not statistically different (One-Way ANOVA, $p=0.09$ and $p=0.77$ for fMRI run 1 193 and 2, respectively, and $p=0.35$ and $p=0.51$ for $1 \mathrm{H}$-fMRS run 1 and 2 , respectively) for rest, PF 194 and UF epochs (ranging, on average, between $360 \mathrm{~ms}$ and $460 \mathrm{~ms}$ ), both during fMRI (Figure 195 1-Figure Supplement 3) and 1H-fMRS (Figure 3-Figure Supplement 3), confirming high 196 and similar levels of subject's attention across conditions. There was not significant correlation 197 between mean pupil diameter (two-tailed t-test, $\mathrm{q}_{\mathrm{FDR}}=1$ and $\mathrm{q}_{\mathrm{FDR}}>0.16$ for fMRI run 1 and 2 , 198 respectively, and $\mathrm{q}_{\mathrm{FDR}}>0.14$ and $\mathrm{q}_{\mathrm{FDR}}>0.88$ for $1 \mathrm{H}-\mathrm{fMRS}$ run 1 and 2 , respectively) or gaze 199 displacement (two-tailed t-test, $\mathrm{q}_{\mathrm{FDR}}=1$ and $\mathrm{q}_{\mathrm{FDR}}>0.59$ for fMRI run 1 and 2, respectively, and $200 \mathrm{q}_{\mathrm{FDR}}>0.13$ and $\mathrm{q}_{\mathrm{FDR}}=1$ for $1 \mathrm{H}$-fMRS run 1 and 2 , respectively) and task performance during both 201 fMRI (Figure 1-Figure Supplement 4) and 1H-fMRS (Figure 3-Figure Supplement 4). 202 Finally, in-scanner head motion during fMRI scans was minimal and not significantly different for 203 all subjects across epochs (mean framewise displacement $0.25 \pm 0.12 \mathrm{~mm}$ for rest, $0.24 \pm 0.14$ $204 \mathrm{~mm}$ for PF, $0.23 \pm 0.08 \mathrm{~mm}$ for UF; One-Way ANOVA, $\mathrm{p}=0.77$ ). Overall, behavioral and 205 physiological variables associated with attentional load were maintained at considerably 206 constant levels in all subjects. 
208 To achieve the same BOLD response in V1 during PF and UF, we reduced the stimulation 209 contrast for the $7.5 \mathrm{~Hz}$ condition to $75 \%$ relative to the $30 \mathrm{~Hz}$ condition (Figure 1-Figure 210 Supplement 5). As expected, we found that the average BOLD time course (One-way ANOVA, $211 \mathrm{p}=0.42$ ), onset time (One-way ANOVA, $\mathrm{p}=0.93$ ) and time-to-peak (paired sample t-test, $\mathrm{p}=0.29$ ) 212 did not differ between PF and UF (Figure 1A,C,D). Similarly, the BOLD change in the subject213 matched spectroscopic VOI (on average consisting of $47 \pm 9 \%$ of BA17, $21 \pm 12 \%$ of BA18, and $21416 \pm 9 \%$ of BA19), was not different $(0.44 \pm 0.30 \%$ for PF versus $0.41 \pm 0.25 \%$ for UF, paired 215 sample t-test, $p=0.71$ ) between the two conditions (Figure 1B). Bayesian analysis indicated 216 moderate evidence for the absence of a difference between conditions (paired sample Bayesian 217 t-test, BF01=3.2). The fMRI activations to PF and UF both peaked in V1 and distinctly spanned 218 bilaterally in secondary visual areas (Figure 2A,B , one-sample t-test, FDR-corrected at 219 cluster level, $q<0.05$, voxel level $p<0.001)$.

220 Different BOLD responses in secondary visual areas to perceived and unperceived 221 flickering

222 To better characterize the effect of the two different stimulations, we estimated the main effect of 223 the flickering frequency. The main effect of PF appeared in the lateral occipital cortices, but not 224 in V1 (Figure 2C,D ; Figure 2-Source Data 1). Bilaterally, 17.5\% (i.e., 18 voxels out of 225 103) of the cortical volume preferentially activated by PF overlapped with the average 226 spectroscopic VOI (Figure 2-Figure supplement $\quad$ 1), corresponding to a contamination of 227 approximately $0.5 \%$ (i.e., 18 voxels out of 3691 ). Although the electrophysiological activity in V1 228 could not be directly assessed in our experiment, based on the literature (Logothetis et al. 2001) 229 we can obtain a rough surrogate of $\mathrm{V} 1$ output by evaluating fMRI signals in the secondary visual 230 areas, which receive input directly from $\mathrm{V} 1$. While the average BOLD response in V1 
231 (Brodmann Area 17) was similar $(0.85 \pm 0.45 \%$ versus $0.80 \pm 0.42 \%$, paired sample t-test, $p=0.72$ )

232 for the two stimulation conditions (Figure 1E), the average BOLD change in secondary visual 233 areas (Brodmann Areas 18 and 19$)$ was significantly $(0.61 \pm 0.29 \%$ versus $0.39 \pm 0.18 \%$, paired 234 sample t-test, $\mathrm{p}=0.008$ ) higher during PF than UF (Figure 1F), indicating a larger output from V1 235 during PF compared with UF. Thus, V1 exhibited the same BOLD signal despite known 236 differences in visual processing for PF and UF (Viswanathan and Freeman 2007).

\section{Dissociation between metabolic and BOLD responses in V1 during loss of visual}

238 perception

239 To assess whether metabolism was sensitive to stimulus processing within V1 we performed 240 single-voxel $1 \mathrm{H}$-fMRS. The reproducible BOLD response in the occipital lobe elicited by the PF 241 and UF stimulations allowed a very accurate VOI positioning for $1 \mathrm{H}$-fMRS. High quality (water 242 linewidth $7.2 \pm 0.6 \mathrm{~Hz}$ ) and artifact-free spectra were reliably obtained from almost all subjects 243 (Figure 3A; Figure 3-Figure Supplement 5; Figure 3-Source Data 1). Compared 244 with resting conditions, the lactate and glutamate concentrations within V1 increased by $2450.29 \pm 0.18 \mu \mathrm{mol} / \mathrm{g}$ and $0.28 \pm 0.16 \mu \mathrm{mol} / \mathrm{g}$, respectively, during the PF stimulation corresponding 246 to an increase of about $28 \%$ and $3 \%$, respectively, over the baseline (paired sample t-test, $\left.247 \mathrm{q}_{\mathrm{FDR}}=0.001\right)$, whereas they both remained at their basal levels $\left(-0.04 \pm 0.13 \mu \mathrm{mol} / \mathrm{g}, \mathrm{q}_{\mathrm{FDR}}=0.42\right.$ for 248 lactate, and $0.03 \pm 0.17 \mu \mathrm{mol} / \mathrm{g}, \mathrm{q}_{\mathrm{FDR}}=0.63$ for glutamate) during the UF stimulation (Figure 249 3B). The lactate and glutamate responses were significantly different (paired sample t-test, $250 \mathrm{q}_{\mathrm{FDR}}=0.01$ for lactate and $\mathrm{q}_{\mathrm{FDR}}=0.003$ for glutamate) among the two stimulation conditions 251 (Figure 3 3B). No other metabolites among those quantified showed a reliable stimulation252 dependent change (Figure 3 -Source Data 2). We were unable to detect a reliable change 253 for aspartate (paired sample t-test, $\mathrm{q}_{\mathrm{FDR}}=0.98$ ), a proposed index of malate-aspartate shuttle 254 and oxidative metabolism (Mangia et al. 2007a). 
255 To confirm our fMRI result of similar BOLD effect during PF and UF, we examined linewidth 256 narrowing of total creatine $(\mathrm{tCr})$ and total $\mathrm{N}$-acetylaspartate (tNAA) signals. We found a 257 stimulation-induced decrease of $\mathrm{tCr}$ and tNAA linewidth during both PF and UF (Figure $3 C, \mathrm{D})$ 258 that was not statistically different between the two conditions $(0.56 \pm 0.04 \mathrm{~Hz}$ vs $0.56 \pm 0.05 \mathrm{~Hz}$ 259 for $\mathrm{tCr}$, paired sample t-test, $\mathrm{p}=0.70 ; 0.55 \pm 0.03 \mathrm{~Hz}$ vs $0.56 \pm 0.05 \mathrm{~Hz}$ for tNAA, paired sample t260 test, $p=0.89)$.

261 To substantiate our $1 \mathrm{H}$-fMRS result, we determined the difference spectra between conditions, 262 which mainly consisted of uncorrelated noise and only a few correlated residuals (Figure $3 E$ ). 263 Specifically, the difference between PF and rest spectra showed a signal in the region 264 corresponding to lactate, and in spectral regions corresponding to glutamate; both signals were 265 absent in the difference between UF and rest spectra. The difference spectra also featured 266 some narrow peaks corresponding to the main singlets of the spectrum, particularly $\mathrm{tCr}$ and 267 tNAA, as a result of BOLD-induced line narrowing (Zhu and Chen 2001). Similar residuals on $268 \mathrm{tCr}$ and tNAA were recognizable in the difference spectrum between UF and rest, but not in the 269 difference spectrum between the two active conditions, again consistent with the evidence of a 270 similar BOLD effect on spectral linewidth elicited by either of the stimulations. Overall, the only 271 correlated signals that survived in the difference spectrum between PF and UF were lactate and 272 glutamate, which strongly supports the significance of the concentration changes based on 273 LCModel quantifications. 


\section{Discussion}

276 The cortical gray matter of the brain features one of the highest metabolic rates of all organ

277 tissues of the human body. Although energy is recognized as a limiting factor for the human

278 cerebral cortex (Herculano-Houzel 2011; Niven and Laughlin 2008), the increase in lactate

279 concentration occurring upon sensory stimulation is not the result of limited oxygen availability

280 (Aalling et al. 2018; DiNuzzo 2016), as it is for skeletal muscle. Why the cerebral cortex

281 upregulates glycolytic metabolism for sensory information processing is unknown, but it is well-

282 established that glycolysis serves specific neurophysiological and neurobiological purposes,

283 such as axonal vesicle transport, vesicle recycling, action potential waveform modulation,

284 reuptake of neuroactive compounds, and dendritic spine remodeling (reviewed in (DiNuzzo and

285 Nedergaard 2017)). Furthermore, lactate is known to be implicated in cognitive processes 286 occurring during waking activity, like learning and memory (Descalzi et al. 2019; Newman et al.

287 2011; Scavuzzo et al. 2020; Suzuki et al. 2011), although the exact underlying mechanisms are 288 still debated (Dienel 2019b; Steinman et al. 2016).

289 In the present study, we asked whether alterations in visual perception are also reflected in 290 metabolic changes within the primary visual cortex in humans. We report that stimulus 291 perception affects the lactate and glutamate response in V1. The PF and UF stimulations 292 elicited, by experimental design, the same average BOLD signals increase inside V1, indicating 293 an equivalent degree of neurovascular coupling and possibly of local synaptic activity in the two 294 experimental conditions (Logothetis 2008). Yet, we observed a significant increase in the 295 regional lactate level only during the PF stimulus, with no appreciable change of lactate during 296 the UF stimulus compared with resting conditions. A temporal dissociation between BOLD and 297 lactate changes has been previously reported during repeated photic stimulations (Mangia et al. 298 2007b). In that case, BOLD response was preserved, but not the lactate accumulation, possibly 
299 due to habituation of neuronal firing. Metabolic adaptation, in terms of glutamate levels, in the 300 presence of constant BOLD and electrophysiological activity was also reported in epilepsy 301 (Peca et al. 2010). These results suggest that the physiological mechanisms underlying BOLD 302 signals and energy metabolism do not necessarily overlap under all experimental conditions.

303 Our results suggest that lactate and glutamate may be dissociated from BOLD changes when 304 cortical input and output are differentially modulated by e.g., intracortical (Viswanathan and 305 Freeman 2007) or thalamocortical (Min et al. 2020) inhibition. In fact, an overall switch toward 306 inhibition is expected to reduce the energy request of the brain, thus impacting on metabolic 307 rates independently of BOLD response (Mangia et al. 2009). Although inhibition, in terms of 308 GABA concentration, has been positively correlated with changes of the BOLD signal (Chen et 309 al. 2005; Donahue et al. 2014; Muthukumaraswamy et al. 2009; Northoff et al. 2007), the 310 dependence of GABAergic neurotransmission and corresponding BOLD changes upon 311 temporal flickering frequency is difficult to predict, as the gating of sensory information is much 312 likely supported by dishinibitory mechanisms (e.g., through vasoactive intestinal peptide-positive 313 interneurons) (Askew et al. 2019; Gasselin et al. 2021; Pi et al. 2013; Williams and Holtmaat 314 2019; Yu et al. 2019). Accordingly, changes in excitation/inhibition equilibrium have been 315 proposed as a source of variability of the $\mathrm{CBF} / \mathrm{CMRO}_{2}$ coupling ratio (Buxton et al. 2014). In 316 more general terms, the metabolic response seems capable of differentiating neural states that 317 are intrinsically distinct, although they induce the same BOLD response (Moradi et al. 2012). 318 Similar BOLD signal changes in correspondence to substantially different alterations in tissue 319 lactate and glutamate level during visual flickering could not be predicted a priori unless 320 assuming fundamental differences in information processing during stimulation. In particular, the 321 firing rate of layer IV neurons that receive input from LGN is higher during UF than PF, as 322 evidenced by the synchronization of these neurons to the stimulus frequency (Gur and 
323 Snodderly 1997; Herrmann 2001; Norcia et al. 2015; Pastor et al. 2003; Regan 1989; Vialatte et 324 al. 2010), yet we found that lactate and glutamate increases during PF, not UF. These findings 325 indicate that the stimulation-induced effect on metabolite concentrations is happening 326 downstream of the input stage in layer IV, and specifically during the intracortical processing 327 involving output layers II/III and V. This conclusion is supported by the fact that high-frequency 328 flickering $(30-60 \mathrm{~Hz})$ abolished MUA, a measure of neuronal spiking (putatively from layer II/III) 329 (Viswanathan and Freeman 2007), but not single neuron recordings from layer IV (Gur and 330 Snodderly 1997).

331 Using compartmentalized metabolic modeling, we have recently obtained evidence that the 332 above-mentioned outcome can be explained by distinct signaling mechanisms underlying 333 spiking and synaptic activity (e.g., pre- and postsynaptic ionic currents) that are indeed 334 frequency-dependent (DiNuzzo and Giove 2012; DiNuzzo et al. 2014). We previously reported 335 that chromatic and achromatic flickering at the same temporal frequency elicited the same 336 neurochemical response in V1 despite differences in the responding neuronal populations (i.e., 337 blob versus interblob) (Bednarik et al. 2018). Together with the results that we report here, this 338 observation suggests that lactate and glutamate, and hence aerobic glycolysis, are both 339 sensitive to cortical processing (e.g., input/output or excitation/inhibition balance) rather than the 340 targeted neuronal population. Our results can be interpreted in keeping with the concept that 341 increased glutamate levels reflect an upregulation of the malate-aspartate shuttle (MAS) 342 (Mangia et al. 2012), although we were unable to report significant differences in aspartate 343 levels likely due to insufficient sensitivity of MRS at 3T. Indeed, the decrease in aspartate during 344 sensory stimulation is commonly observed at 7T, but to our knowledge has never been reported 345 at 3T, most likely due to limited sensitivity of detection. In fact, the small absolute concentration 346 of aspartate, its multiplet spectrum profile, and its overlap with other metabolites pose several 
347 challenges for detection of the small changes (around $0.1-0.2 \mathrm{mmol} / \mathrm{L}$ ) occurring during a 348 functional design. Similar considerations actually apply to glucose, which also was not reported 349 in this study to decrease as observed in 7T studies (Mangia et al. 2007a). The NADH produced 350 by the metabolism of glucose to lactate has to be transported from the cytosol to the 351 mitochondria to be used for energy production within the electron transport chain. Among other 352 components of the MAS, such process requires that glutamate is transported from cytosol to 353 mitochondria and concomitantly aspartate is counter-transported from mitochondria to cytosol 354 through the aspartate-glutamate carrier (AGC1/Aralar). The AGC1/Aralar carrier-mediated 355 transport is a rate-limiting step within the MAS and therefore it lags behind the concomitant 356 increase in tricarboxylic acids (TCA) cycle and NAD ${ }^{+} / \mathrm{NADH}-$ redox shuttling in mitochondria. In 357 agreement with existing literature (Hertz and Chen 2017; Hertz and Rothman 2017; Lin et al. 358 2012; Mangia et al. 2012; Mangia et al. 2007a), we interpret the increase in glutamate (and 359 decrease in aspartate) concentration as reflecting an increase in the rate of MAS, with 360 accumulation of glutamate at the cytosolic side and reduction of aspartate at the mitochondrial 361 side. Notably, the MAS does not necessarily correlate with mitochondrial respiration and 362 cerebral blood flow, as evidenced by the findings that (i) oxidative metabolism and $\mathrm{CMRO}_{2}$ are 363 enhanced at both low and high flickering frequencies (Viswanathan and Freeman 2007), and (ii) 364 glutamate and aspartate, but not lactate, correlate with BOLD signals during low-frequency ( 8 $365 \mathrm{~Hz}$ ) visual stimulation (Bednarik et al. 2018; Ip et al. 2017). To what extent aerobic glycolysis 366 and MAS can dissociate from oxidative phosphorylation in the brain remains to be established, 367 but previously published data strongly indicates that the increase in lactate and glutamate levels 368 is the consequence of the surge in glycolysis and the ensuing increase of cytosolic NADH:NAD ${ }^{+}$ 369 ratio taking place during neuronal activation (Diaz-Garcia et al. 2017). 
370 While the similar BOLD response in V1 is a direct consequence of our experimental design, we 371 additionally found that secondary visual areas were recruited to a larger extent during PF 372 stimulus than UF stimulus, supporting the argument that their activation is necessary for 373 perception (Lamme et al. 2000; Salminen-Vaparanta et al. 2019; Tong 2003). The preferential 374 response of secondary visual areas to perceived stimuli that we observe here broadly confirms 375 previous results of a strong BOLD activity in ventral ("visual-for-perception" processing) and 376 dorsal ("visual-for-action" processing) streams during visible stimuli and a reduction of their 377 activity in conditions of invisible stimulation (Hesselmann and Malach 2011). However, since our 378 stimulation protocol is associated by design with higher activation of output layers of $\mathrm{V} 1$ and 379 hence higher input to V2/V3, we cannot conclusively and unambiguously establish that the 380 higher activation of secondary visual areas is causally linked to perception. In fact, the very 381 notion that BOLD activity in secondary visual areas actually reflects $\mathrm{V} 1$ output is uncertain 382 because we have no direct measurement to firmly support such assumption, although it is very 383 tightly linked to the unique architecture of the visual system.

384 The role of the primary visual cortex in stimulus perception is an intense area of research (Gail 385 et al. 2004; Leopold and Logothetis 1996; Maier et al. 2008; Polonsky et al. 2000; Wunderlich et 386 al. 2005). Previous fMRI studies investigating the dependence of V1 BOLD activity on visual 387 perception produced controversial results, which might be related to the difficulty of 388 disentangling perception from spatial attention. Indeed, it has been shown that attention, but not 389 perception, modulates the BOLD signal in human V1 (Watanabe et al. 2011). In fact, perception 390 was found to enhance the BOLD response within V1 for specific experimental designs (e.g., 391 flash suppression of perception) (Yuval-Greenberg and Heeger 2013). Our study employed a 392 substantially different stimulation paradigm that specifically allowed us, by adjusting the 393 stimulation contrast, to induce the same BOLD response, and inductively an equivalent mean 
394 degree of synaptic activity, within V1 under the two experimental conditions (Logothetis 2008). 395 Most importantly, we did not use any additional stimulus (e.g., visual masking) to suppress or 396 modulate visual perception. On the contrary, we used a simple way to modulate perception for 397 extended periods of time (required by $1 \mathrm{H}$-fMRS) while maintaining attention at a nearly constant 398 level, as evidenced by pupillometry and task performance. In particular, we employed the well399 known dependence of BOLD response in V1 to flickering, which at full-contrast peaks at 4-8 Hz 400 and settles around $70 \%$ of its maximal value even at frequencies above $30 \mathrm{~Hz}$ (Thomas and 401 Menon 1998), i.e., in the absence of flickering perception. Previous literature reported that the 402 peak activations in V1 and secondary visual areas are stimulation-dependent and occur at 403 distinct temporal frequencies (4 Hz or $8 \mathrm{~Hz}$ in V1, and $20 \mathrm{~Hz}$ or $40 \mathrm{~Hz}$ in secondary visual areas 404 for chromatic or luminance flickering, respectively) (Chai et al. 2019; Fox et al. 1986; Kastner et 405 al. 2004; Kwong et al. 1992; Singh et al. 2000). Interestingly, the preferred frequency of 406 secondary visual areas is just below the CFF for chromatic flickering $(\sim 25 \mathrm{~Hz})$ and luminance 407 flickering $(\sim 50 \mathrm{~Hz})$ (Shady et al. 2004). In our experiments, the isoluminant chromatic flickering 408 stimulation at $30 \mathrm{~Hz}$ is above the CFF and accordingly we found that the BOLD response in 409 secondary visual areas drops substantially compared with the concurrent BOLD response in V1. 410 Overall, by using visual stimulations below and above the CFF and adjusting the contrast of the 411 low-frequency stimulus we were able to modulate perception alongside BOLD activity in 412 secondary visual areas with a unchanged BOLD activity in V1. It should be noted that subtle 413 differences in the qualitative nature of the BOLD signal response might exist between the two 414 conditions, although the trends towards longer onset time and time-to-peak during UF compared 415 with PF were not statistically significant.

416 Our study has some limitations. For instance, the fMRI measurements have been obtained 417 using 30 s epochs, while the fMRS measurements have been obtained using 4 min epochs. 
418 Long acquisition times are required for achieving a good sensitivity of the $1 \mathrm{H}$-fMRS 419 measurements and a reliable metabolite quantification, even at magnetic fields of 3T. 420 Nonetheless, results in both humans (Mangia et al. 2007b) and rats (Just et al. 2013) have 421 shown that prolonged (i.e., minutes) stimulations elicit a persistent BOLD response with minimal 422 attenuation. Although we cannot exclude a certain degree of neuronal adaptation, we confirmed 423 that the BOLD effect during the fMRS acquisition was indeed relatively stable as determined by 424 the linewidth changes of $\mathrm{tCr}$ and tNAA signals. Another limitation to consider is represented by 425 the large volume used for $1 \mathrm{H}$-fMRS measurements. It could be possible that the observed 426 changes in lactate levels include distinct neuronal populations showing non uniform responses. 427 Indeed, although the main differential effect of frequency was located far from the calcarine 428 sulcus, it also involved some mid hemispheric areas presumably included in the spectroscopic 429 voxel. As an illustration, heterogeneity in the response to stimulation within V1 might be due to, 430 e.g., eccentricity (Chai et al. 2019), which we however minimized by using a $3^{\circ}$ foveal 431 stimulation. There is also an hemispheric lateralization for the spatial frequencies (Kauffmann et 432 al. 2014), but this is not likely to apply to our study, as we used the same radial checkerboard 433 (i.e., including many spatial frequencies) for both PF and UF. The finding that the changes in 434 BOLD signal during PF and UF were nearly double in the anatomical (i.e., Brodmann atlas435 based) V1 compared with the spectroscopic VOI indicates a substantial partial-volume effect. 436 The associated loss of sensitivity entails that the lactate concentration change that we report 437 here might in fact be under-estimated. Furthermore, we cannot exclude that feedback input to $438 \quad$ V1 from secondary visual areas might be taking place during stimulus perception (Watkins et al. 439 2006). However, under our experimental design we were able to fully control BOLD response in 440 V1 by only acting on stimulus contrast, without altering the subjective perception of the stimuli, 441 which indicates that the BOLD activity in V1 largely reflected stimulus features. Finally, we 442 based the positioning of the spectroscopic VOI solely on the online estimate of the stimulus- 
443 induced BOLD signal change. In particular, we could not perform online retinotopic mapping for

444 driving the positioning of the spectroscopic VOI, as this would have increased the acquisition

445 protocol duration to an impractical length for guaranteeing good attention levels and absence of

446 motion during the $1 \mathrm{H}$-fMRS session. In any case, partial-volume effects are unavoidable

447 because the STEAM sequence we used requires a box-shaped VOI, which would have not

448 allowed us to entirely avoid the inclusion of tissue outside $\mathrm{V} 1$, regardless of the availability of the 449 retinotopic map.

451 Conclusion

452 In this study we report for the first time that visual perception has a measurable metabolic effect 453 on V1. Although we could not establish any causal relation between metabolism and perception, 454 our findings imply that BOLD fMRI and 1H-fMRS are complementary techniques. Specifically, 455 they are capable of highlighting different aspects of neural activation and stimulus processing, 456 e.g., under conditions in which synaptic and spiking activity are partially disentangled due to an 457 alteration of excitation/inhibition patterns and, in turn, local input/output balance.

458 Although we focused on the visual pathways, it is conceivable that our results can be translated 459 to other sensory modalities. For example, tactile (Laureys et al. 2002) or auditory (Boly et al. 460 2004; Laureys et al. 2000) stimulations during vegetative state can still activate primary 461 somatosensory cortex (S1) or primary auditory cortex (A1), respectively, in the absence of 462 perception and without the involvement of higher-order associative areas.

463 We suggest that the cortical metabolic profile might be an indicator of sensory perception, in 464 keeping with the dynamics of lactate and glutamate across the sleep-wake cycle (Aalling et al. 465 2018; DiNuzzo and Nedergaard 2017; Naylor et al. 2012; Naylor et al. 2011) as well as with the 
466 relation between lactate and arousal (Zuend et al. 2020). Elevated brain lactate and glutamate 467 levels are associated with wakefulness and memory formation, which naturally require the 468 processing of incoming sensory stimuli, like the control exerted by the central visual pathways 469 for either gating or filtering out behaviorally relevant or irrelevant visual information. As such, the 470 metabolic response to perceived, but not unperceived, sensory stimulation could be enabling 471 factors for learning and memory, as indicated by the relevance of aerobic glycolysis and lactate 472 in synaptic plasticity mechanisms (Bueschke et al. 2021; Descalzi et al. 2019; DiNuzzo 2016; 473 Harris et al. 2019; Herrera-López et al. 2020; Jourdain et al. 2018; Kobayashi et al. 2019; 474 Lundquist et al. 2021; Margineanu et al. 2018; Scavuzzo et al. 2020; Wang et al. 2019; Yang et 475 al. 2014). In particular, aerobic glycolysis and lactate might reflect cortical information 476 processing and, in turn, intracortical communication, in agreement with the relation between 477 regional metabolic rates of glucose utilization and resting-state network dynamics in the cerebral 478 cortex (Jamadar et al. 2021; Noack et al. 2017; Spetsieris et al. 2015; Su et al. 2018; Thompson 479 2018). 


\section{$481 \quad$ Materials and methods}

\section{Setup}

483 MR measurements were performed on a $3 T$ head-only scanner (Siemens Allegra, Erlangen, 484 Germany), equipped with high performance gradients (amplitude $40 \mathrm{mT} / \mathrm{m}$, rise time $100 \mu \mathrm{s}$ ). A 485 custom-built quadrature surface coil (loop and butterfly design, Rapid Biomedical, Rimpar, 486 Germany) was used for both RF transmission and detection. The coil design traded usable 487 volume (Figure S5) for peak sensitivity. For imaging, first and second order shim terms were 488 adjusted using the automatized Siemens routine based on field map acquisition and fitting. For 489 MRS, shimming was optimized using FASTMAP with EPI readout (Gruetter and Tkác 2000) and 490 manually refined when necessary to reach a water linewidth of less than $9 \mathrm{~Hz}$.

\section{Subjects}

492 A total of 32 healthy volunteers were initially enrolled for this study after they gave informed 493 consent, according to the Helsinki declaration and to European Union regulations, and following 494 the approval by the local Ethics Committee of the Fondazione Santa Lucia IRCCS (Rome). 495 Exclusion criteria included any kind of previous neurological or psychiatric disease and impaired 496 visual acuity. Thirteen subjects were discarded either upon online fMRI processing (see below) 497 or in early quality control on data, because of motion (3) or because the most activated area 498 was an unfavorable position with respect to the surface-coil sensitive volume (10). Nineteen 499 healthy volunteers (10 females, 9 males; age $25 \pm 4$ years, mean $\pm S D$; age range 20 to 35 years) 500 were thus considered for this study. Sample size calculations performed before the study 501 assumed a two-tail paired t-test design, a power of 0.95 and were based on an effect magnitude 502 for lactate change (photic stimulation vs rest) of $0.20 \pm 0.15 \mu \mathrm{mol} / \mathrm{g}$ as estimated in our previous 503 works at 7T (Bednarik et al. 2018). Reduced sensitivity of 3T vs 7T was empirically accounted 504 for by larger VOI and extended data averaging (144 transients per condition, $10 \mathrm{ml} \mathrm{VOI} \mathrm{at} \mathrm{3T} \mathrm{vs}$ 
50564 transients per condition, $8 \mathrm{ml} \mathrm{VOI}$ at $7 \mathrm{~T}$ ). The resulting required sample size of 10 was 506 roughly doubled to account for multiple comparisons.

\section{Visual stimulation}

508 Visual stimulation consisted in a radial checkerboard flickering either at $7.5 \mathrm{~Hz}$ (perceived

509 flickering stimulation, PF) or at $30 \mathrm{~Hz}$ (unperceived flickering stimulation, UF). The alternating 510 frames included either a gray-green or a gray-red checkerboard. The stimuli were programmed

511 in Cogent 2000 version 1.29 working under Matlab 2006b (The Mathworks, Natick, MA, USA).

512 and delivered using an MR-compatible fMRI system with stereo 3D goggles (VisuaStim Digital, 513 Resonance Technology, Inc., Northridge, California, USA). Subjects were fitted with the 514 VisuaStim video goggles (Resolution: SXGA 1280x1024 pixels, Refresh Rate: 60Hz, Field of

515 View: 30 degrees Horizontal x 24 degrees Vertical, White Luminance: $70 \mathrm{~cd} / \mathrm{m} 2$ max, Contrast 516 Ratio: intrinsic 100:1 measured per VESA FPDM Standard).

\section{Experimental protocol}

518 Visual stimulations were presented in eight (fMRI) or four (fMRS) epochs, with stimulation 519 epochs (either PF or UF) interleaved by rest (isoluminant, uniform gray images) epochs. 520 Since the CFF is higher for luminance than for chromatic flickering (Jiang et al. 2007), before 521 each session the brightness of green squares during the UF condition (i.e. isoluminant 522 condition) was adjusted interactively by the subject, who piloted increasing and decreasing 523 brightness ramps and was instructed to identify the brightness level corresponding to loss of 524 luminance flickering perception of the resulting yellow. The green level was then set midway 525 between the two perceptual vanishing levels. The stimulus contrast was adjusted in preliminary 526 acquisitions on five subjects, in order to induce a comparable BOLD response to PF and UF in 527 V1 (Figure $\quad 3 A$ ). During the fMRI sessions epochs lasted 30 seconds each (total 4 minutes), 528 while for fMRS acquisitions epochs were 4 minutes long (total 16 minutes). Each subject 
529 underwent $1 \mathrm{fMRI}$ and 2 fMRS runs (10 minutes apart, without moving the subject from inside

530 the scanner); the order of PF and UF conditions was counterbalanced within each subject, and

531 the initial stimulation type was randomized between subjects.

\section{Task}

533 In order to maintain visual fixation and keep a constant attentional state, the subjects were

534 asked to focus on a central target (a cross) and to press a button whenever the target rotated.

535 Subjects were specifically instructed to maintain their attention on the fixation cross rather than

536 focusing on reaction times (i.e., speed to push the button). The number of rotations was

537 constant across the different epochs (3 for each fMRI epoch and 24 for each $1 \mathrm{H}-\mathrm{fMRS}$ epoch,

538 or approx. 1 rotation every 10 seconds), while the exact timing of the rotation was pseudo-

539 randomized (range 2-18 seconds).

\section{Anatomical and fMRI data acquisition}

541 Each study started with an anatomical acquisition (MPRAGE T1-weighted volumetric scan,

542 resolution $1.2 \times 1.2 \times 1.2 \mathrm{~mm}^{3}$, para--axial slices, in-plane FOV $190 \times 70 \mathrm{~mm}^{2}$, TE=4.38 ms,

$543 \mathrm{TR}=2000 \mathrm{~ms}, \mathrm{Tl}=910 \mathrm{~ms}, \mathrm{FA}=8^{\circ}$ ). Then, one $\mathrm{fMRI}$ session (pseudo-randomized order of

544 stimulation across subjects) was acquired (gradient echo with EPI readout, resolution

$5452.2 \times 2.2 \times 2.2 \mathrm{~mm}^{3}, 26$ para--axial contiguous slices, FOV $190 \times 70 \mathrm{~mm}^{2}, \mathrm{TE}=30 \mathrm{~ms}, \mathrm{TR}=1500 \mathrm{~ms}$,

$546 \mathrm{FA}=70^{\circ}$ ). $\mathrm{fMRI}$ scans were processed online for subsequent MRS voxel positioning (online

547 processing included motion correction, smoothing, cross-correlation with a square-wave model;

548 the two scans following each condition change were discarded in order to reduce the effects of

549 BOLD signal transients. Online processing was discarded after voxel positioning).

550 To confirm the absence of any detectable brain pathology in our subjects, T1 and T2 weighted

551 anatomical scans were acquired with a standard volume birdcage coil after the end of the 
552 functional scans. Anatomical scans included an MPRAGE acquisition (resolution 1.0×1.0×1.0

$553 \mathrm{~mm}^{3}$, para-axial slices, in-plane FOV $256 \times 160 \mathrm{~mm}^{2}, \mathrm{TE}=2.48 \mathrm{~ms}, \mathrm{TR}=2150 \mathrm{~ms}, \mathrm{TI}=1000 \mathrm{~ms}$,

$554 \quad F A=8^{\circ}$ ), that was later used during the post-processing for normalization purposes.

\section{5 fMRS data acquisition}

556 The spectroscopic voxel (size $25 \times 20 \times 20 \mathrm{~mm}^{3}$ ) was localized in the most activated area within

$557 \mathrm{~V} 1$, based on both anatomical scan and results of the online fMRI processing. The voxel was

558 located either left or right of the interhemispheric fissure to minimize the cerebrospinal fluid

559 fraction in the VOI. Two MRS sessions were acquired with an optimized, in-house written

560 STEAM sequence $\left(\mathrm{TE}=7 \mathrm{~ms}, \mathrm{TM}=50 \mathrm{~ms}, \mathrm{TR}=3000 \mathrm{~ms}, \mathrm{FA}=70^{\circ}\right)$ which included outer volume

561 saturation and VAPOR water suppression (Tkác et al. 2001; Tkác et al. 1999). An eight-step

562 phase cycle was used; transients were averaged within each phase cycle, and each phase

563 cycle was saved separately for further processing. Water unsuppressed data were acquired

564 from the same voxel for eddy currents compensation (Klose 1990). In order to minimize T1

565 weighting, the flip angle was kept below the calculated Ernst angle in both fMRI and fMRS

566 acquisitions.

\section{Pupillometry}

568 In order to monitor attentional state with a physiological parameter, we acquired pupillometry 569 data using an eye-tracking system (Applied Science Laboratories, model 504) equipped with a 570 remote pan/tilt optic infrared module and a video camera that was custom-adapted for use in the

571 scanner. Subject gaze position and pupil size data were processed as previously described 572 (DiNuzzo et al. 2019). 


\section{3 fMRI data processing}

574 fMRI (offline) processing was performed with routines from SPM12 (Wellcome Trust Centre for

575 Neuroimaging, UCL) working under Matlab 2018b, AFNI (Cox 1996) and FSL5 (Jenkinson et al.

576 2012), and with custom Matlab routines. FMRI data were realigned to their mean image to

577 compensate for head movements. Realigned images were then normalized to the MNI template

$578(2 \times 2 \times 2 \mathrm{~mm} 3)$ by using the non linear transformation calculated on the MPRAGE acquired with

579 the volume coil, after a linear co-registration that used the surface-coil MPRAGE image as

580 intermediate step to best match the volume-coil MPRAGE to the fMRI series. Normalized

581 images were spatially smoothed with an isotropic $4 \mathrm{~mm}$ full width at half-maximum (FWHM)

582 Gaussian kernel. A general linear model (GLM) analysis with boxcars functions convolved with

583 the hemodynamic response function was applied to localize the regions responding to the PF

584 and UF conditions. The GLM model included a high-pass filter $(128 \mathrm{~Hz})$ and the 6 rotational and

585 translational parameters obtained from the realignment step.

586 For the volume-of-interest (VOI) based analysis, images underwent the same processing

587 pipeline with the only exception that the analysis was performed in the subject-specific space,

588 thus, no spatial normalization step was applied.

589 Head motion during $\mathrm{fMRI}$ acquisitions was evaluated using the framewise displacement, which

590 was calculated as the L1-norm of the realignment-derived parameters after converting angles to 591 linear displacements (Power et al. 2012).

\section{1H-fMRS data processing}

593 MRS data were preprocessed using jMRUI 5.2 (Naressi et al. 2001) and custom Matlab 594 routines. Data were corrected for residual eddy currents, individually phased and frequency 595 shifted to compensate for $\mathrm{B}_{0}$ drifts, and averaged in blocks corresponding to each rest or 
596 stimulation epoch. The first 8 transients of each epoch, i.e. the first full phase cycle $(24 \mathrm{~s})$ were 597 discarded to avoid metabolic transients (Mangia et al. 2007a). Subsequent phase cycles were 598 inspected individually. They consistently showed good water suppression and no trace of lipidic 599 contamination. A few 8-transient spectra (maximum one in each epoch) featured anomalous line 600 broadening, line splitting or otherwise reduced quality, putatively related to subject motion or 601 deep inspiration, and were discarded before averaging. Each epoch spectrum was thus the 602 average of 64-72 transients. The resulting averages were finally quantified using LCModel 6.3-1 603 (Provencher 1993) with a tailored basis set. Basis metabolites included alanine, aspartate (Asp), 604 creatine (Cr), Y-Aminobutyric acid (GABA), glutamine (GIn), glutamate (Glu), glycine, 605 glycerylphosphorylcholine, glutathione (GSH), lactate (Lac), myo-inositol (Ins), N606 acetylaspartate (NAA), N-acetylaspartylglutamate (NAAG), phosphocholine, phosphocreatine, 607 phosphorylethanolamine, scyllo-inositol, and taurine (Tau). Glucose, an important marker of 608 energy metabolism, whose changes have also been reported in previous 7T studies (Bednarik 609 et al. 2015; Mangia et al. 2007a), was not included in the basis set due to highly unreliable 610 quantification observed in preliminary tests. Metabolite spectra were simulated using GAVA 611 (Soher et al. 2007), including information on the sequence pulse program. The basis set 612 included also a subject-specific macromolecular (MM) signal, that was acquired on each subject 613 in the occipital region, using a double inversion recovery approach (STEAM, TI1=1700 ms, $614 \mathrm{TI} 2=520 \mathrm{~ms}, \mathrm{TE}=7 \mathrm{~ms}, \mathrm{TM}=50 \mathrm{~ms}, \mathrm{TR}=2000 \mathrm{~ms}, \mathrm{FA}=90^{\circ}$ ) (de Graaf et al. 2006), that resulted in 615 almost complete metabolite nulling, averaged between subjects, and then modeled with Hankel616 Lanczos singular value decomposition. LCmodel quantifications with Cramér-Rao lower bounds 617 (CRLB) above 30\% were discarded, except for Lac for which the threshold was set at $35 \%$. 618 Since this study is focused on epoch-to-epoch metabolic changes, absolute quantification with 619 water referencing was not performed to avoid the uncertainty associated with between-scans 620 motion, relaxation and partial volume corrections for white matter and gray matter in the VOI. 
621 Metabolites were instead normalized to the $\mathrm{tCr}$ signal amplitude fitted over each run, which was

622 here assumed to correspond to $7.5 \mu \mathrm{mol} / \mathrm{g}$ (see de Graaf, 2007). Eleven metabolites were 623 quantified in at least 15 subjects ( $80 \%$ of participants). These included aspartate, total creatine $624(\mathrm{tCr}), \mathrm{GABA}$, glutamate, glutamine, glutathione, lactate, myo-inositol, total $\mathrm{N}$-acetylaspartate 625 (tNAA, or NAA plus NAAG), total choline, taurine. In order to take BOLD-induced spectral 626 linewidth alterations into account during the fMRS acquisitions, we determined the BOLD effect 627 as the kernel size (in $\mathrm{Hz}$ ) that minimized the difference spectra (windowed around the reference 628 metabolite peak, or 2.82-3.14 ppm for $\mathrm{tCr}$ and $1.75-2.25 \mathrm{ppm}$ for $\mathrm{tNAA}$ ) between the stimulated 629 epoch (either PF or UF) and the preceding resting epoch, under the assumption that the levels 630 (i.e., area under the peak) of $\mathrm{tCr}$ and tNAA remain constant across epochs (de Graaf 2007). All 631 spectra were then averaged according to three categories: rest, PF and UF conditions, and 632 differences were calculated after performing linewidth matching between stimulated and 633 corresponding rest conditions (Mangia et al. 2007a).

\section{Statistics}

635 For pupillometry and task performance results, statistical comparisons were made using 636 Student's t-test and One-Way ANOVA on the rest, UF, and PF conditions. No post-hoc test was 637 necessary. Correlations between mean pupil diameter or gaze displacement and task 638 performance were computed as Kendall's tau coefficients and corrected for multiple 639 comparisons using False Discovery Rate (FDR).

640 For fMRI results, correction for multiple comparisons in functional voxel-based analysis was

641 performed using FDR correction. Resulting clusters were also checked through Monte Carlo 642 Simulation using the AFNI tool Alphasim (Cox 1996) after estimation of residuals smoothness.

643 Comparison between conditions was performed in Matlab 2018b with ANOVA, unpaired, or 644 paired t-test as appropriate. Significance of difference of BOLD response in the spectroscopy 
645 voxel (Figure 1B) was additionally assessed with Bayesian paired t-test with level of evidence 646 set to 3.

647 For fMRS results, statistical analysis was restricted to those reliably quantified metabolites 648 associated with energy metabolism that showed consistent functional changes in previous fMRS

649 studies (Bednarik et al. 2015; Bednarik et al. 2018; Lin et al. 2012), namely Lac, Glu, and Asp. 650 Metabolite concentration changes referred to the corresponding resting epoch and between 651 different active conditions were tested using paired sample t-tests, with FDR correction for 9 652 multiple comparisons.

653 Data were presented as the mean \pm standard deviation (SD). A p-value, or a $\mathrm{q}_{\mathrm{FDR}}$-value where 654 relevant, of less than 0.05 was considered as statistically significant. 
657 The authors wish to thank Edward J. Auerbach for the FASTMAP implementation on Siemens 658 platform, provided by the University of Minnesota under a C2P agreement, and for his help with 659 the setup of offline shim currents calculation. Siemens Healthineers is acknowledged for 660 providing source code and information on the shim coils.

\section{Data and materials availability}

663 All data that support the findings of this study are available from the corresponding author upon 664 signing a MTA that would include a list of authorized researchers and the commitment to no 665 further distribute the materials. Data is not available in a public repository at the time of this 666 publication because of constraints originally set by the Ethics Committee and included in the 667 informed consent signed by participants.

668 The study was developed using SPM12 (https://www.fil.ion.ucl.ac.uk/spm/software/spm12/), 669 LCmodel (http://s-provencher.com/lcmodel.shtml), jMRUI (http://www.jmrui.eu/) and custom 670 scripts. Custom MATLAB code for fMRI and $1 \mathrm{H}$-fMRS data processing is available in figure 671 supplements (Figure 1-Source Data 2 and Figure $\quad 3$-Source Data 3) and in online 672 public repository (https://github.com/dmascali/mni2atlas).

\section{Ethics statement}

675 All experiments with human subjects performed by the authors complied with all applicable 676 ethical standards, including the Helsinki declaration and its amendments, institutional/national 677 research committee standards, and international/national/institutional guidelines. 


\section{Captions}

680

681 Video 1. The movie shows the physiological fluctuations of the pupil diameter, as well as the

682 relevant behavioral responses, of a representative subject during a $\mathrm{fMRI}$ stimulation cycle, 683 which included one UF (30 s) and one PF (30 s) epoch.

685 Figure 1. Main and differential effects of stimulation assessed by VOI-based fMRI analysis. (A) Mean time-course of BOLD signals in the transition between rest and PF or rest 687 and UF, averaged over the fMRI voxels corresponding to the subject-specific spectroscopic VOI. 688 (B) BOLD percent change during the experimental conditions, averaged over the fMRI voxels 689 corresponding to the subject-specific spectroscopic VOI. No statistically significant difference in 690 BOLD response was found between the two conditions with conventional statistics, and 691 Bayesan paired sample t-test indicated moderate evidence for absence of difference between 692 the conditions. (C,D) Average onset time as a function of peak intensity fraction and 693 corresponding time-to-peak (i.e., at 100\% peak intensity). There is a small, although not 694 statistically significant trend for slower onset and longer time-to-peak of BOLD increase during 695 the UF condition. (E) BOLD percent change averaged over the fMRI voxels corresponding to 696 the Brodmann Area 17 (i.e., V1). Within V1, there is no difference between PF and UF condition 697 (unpaired two-sample t-test, $p=0.72$ ). (F) BOLD percent change averaged over the fMRI voxels 698 corresponding to the Brodmann Areas 18 and 19 (e.g., including V2, V3a, V4V, V5/MT). Within 699 these areas, the response to PF is significantly larger than the corresponding response to UF 700 (unpaired two-sample t-test, $p=0.008$ ). 
701 Figure 1 - Figure Supplement 1. Eye position and gaze displacement during fMRI

702 sessions. $(A, B)$ Left panels. Average heatmaps of eyes position (across subjects) during fMRI

703 sessions (Runs 1 and 2, respectively). Middle panels. Stability of mean gaze displacement from

704 the fixation point. Right panels. Gaze displacement was not different across conditions.

705 Figure 1 - Figure Supplement 2. Pupil size dynamics during fMRI sessions. (A,B) Left

706 panels. Average pupil diameter (across-subjects) during fMRI sessions (Runs 1 and 2,

707 respectively). Right panels. Mean pupil diameter was not statistically different across conditions.

708 Figure 1 - Figure Supplement 3. Task performance during fMRI sessions. (A,B) Average

709 task performance (across-subjects) during fMRI sessions (Runs 1 and 2, respectively).

710 Response delay was not statistically different across conditions.

711 Figure 1 - Figure Supplement 4. Correlation of task performance with eye position/pupil

712 size during fMRI sessions. (A,B) Correlation between task performance and eyetracking data

713 during fMRI sessions (Runs 1 and 2, respectively). Left panels. There was no correlation

714 between response delay and mean gaze displacement. Right panels. There was no correlation

715 between response delay and mean pupil diameter.

716 Figure 1 - Figure Supplement 5. PF versus UF BOLD matching. Calibration of image

717 contrast to match BOLD response in V1 to PF and UF obtained in a preliminary session. The

718 contrast of the PF image was reduced to $75 \%$ for subsequent stimulations (i.e. common to all 719 subjects).

720

721

722 
723 Figure 1 - Source Data 1. Demographics and fMRI study parameters.

Figure 1 - Source Data 2. Datasets and Matlab scripts for generating panels from Figure 1

726 and associated figure supplements.

Figure 2. Main and differential effects of stimulation assessed by voxel-based fMRI

analysis. (A) Statistical maps for group-averaged positive effect of the PF visual stimulation versus rest (i.e. PF>rest). (B) Statistical maps for group-averaged positive effect of the PF visual

731 stimulation versus rest (i.e. UF>rest). (C) Overlap between activation maps relative to PF and

732 UF. (D) Differential effect of stimulation (PF>UF). The differential response related to perception

733 is localized in the lateral occipital cortex (secondary visual areas), with no responding voxels

734 inside V1. For comparison, the inverse differential effect of stimulation (UF>PF) has no

735 significant responding voxels (not shown). All statistical activation maps are thresholded at

$736 \mathrm{p}<0.001$, with a FDR correction at the cluster level (corresponding to $\mathrm{q}_{\mathrm{FDR}}<0.05$ ), and overlaid

737 on MNI template.

738 Figure 2 - Figure Supplement 1. Positions of regions and volumes of interest within the

739 imaging field. (A) Intensity map of EPI images acquired with the surface coil normalized to MNI

740 template, superimposed to different ROls (in white). Top panels. Average spectroscopic voxel

741 (1H-fMRS VOI). Bottom panels. BA17 (i.e., V1), BA18, and BA19. (B) Location of the PF>UF

742 regions (green) in comparison with the spectroscopic VOI (red). The overlapping voxels are in

743 yellow. Note that the average spectroscopic VOI has been thresholded to have the same

744 mean volume (i.e., number of voxels) of the single-subjects $1 \mathrm{H}$-fMRS VOIs.

745 Figure 2 - Source Data 1. Cortical regions preferentially activated by PF compared to UF. 
747 Figure 3 . Effects of stimulation on the cortical metabolic profile assessed by ${ }^{1} \mathrm{H}$-fMRS

748 analysis. (A) Spectroscopic data acquired during resting $\quad$ (R, cyan) as well as PF (black) and

749 UF (gray) conditions, averaged across subjects. A single-subject representative voxel location is 750 reproduced on a parasagittal view of the BOLD activation and superimposed on the anatomical

751 scan from the same subject. For visualization purposes, the processing of the spectra included

752 frequency and phase correction of single transients, averaging, eddy currents correction, and

753 Fourier transform. (B) Lactate, glutamate, and aspartate concentration changes during the

754 stimulation conditions, relative to the rest conditions acquired immediately before. Data are

755 averaged across subjects. There is significant increase in lactate $(+28 \%)$ and glutamate $(+3 \%)$

756 levels induced by PF stimulus, but not by UF stimulus. The concentration changes of the two 757 metabolites were significantly different across the stimulation conditions, while there was no 758 change for aspartate. (C,D) Spectral tCr and tNAA linewidth changes induced by the PF and UF 759 stimuli show no statistically significant difference. (E) Differences between spectra acquired in 760 the three experimental conditions. For reference, the corresponding LCModel fits are reported 761 on the bottom for the Lac and Glu signals. tCr and tNAA singlets showed the expected BOLD 762 related features: there is a difference between stimulation and rest, but the difference spectra 763 between the active conditions are within the noise. In the regions of lactate and glutamate the 764 difference spectra between PF and rest and between PF and UF are similar, while they are 765 clearly distinct from the difference spectra between UF and rest.

766 Figure 3 - Figure Supplement 1. Eye position and gaze displacement during 1H-fMRS

767 sessions. (A,B) Left panels. Average heatmaps of eyes position (across subjects) during $1 \mathrm{H}-$

768 fMRS sessions (Runs 1 and 2, respectively). Middle panels. Stability of mean gaze 
displacement from the fixation point. Right panels. Gaze displacement was not different across

770 conditions.

$771 \quad$ Figure $\quad 3$ - Figure Supplement 2. Pupil size dynamics during 1 H-fMRS sessions. $(A, B)$

772 Left panels. Average pupil diameter (across-subjects) during 1H-fMRS sessions (Runs 1 and 2,

773 respectively). Right panels. Mean pupil diameter was not statistically different across conditions.

774 Figure $\quad 3$ - Figure Supplement 3. Task performance during $1 \mathrm{H}$-fMRS sessions. $(A, B)$

775 Average task performance (across-subjects) during $1 \mathrm{H}$-fMRS sessions (Runs 1 and 2,

776 respectively). Response delay was not statistically different across conditions.

777 Figure $\quad 3$ - Figure Supplement 4. Correlation of task performance with eye

778 position/pupil size during fMRI sessions. (A,B) Correlation between task performance and

779 eyetracking data during $1 \mathrm{H}-\mathrm{fMRS}$ sessions (Runs 1 and 2, respectively). Left panels. There was

780 no correlation between response delay and mean gaze displacement. Right panels. There was

781 no correlation between response delay and mean pupil diameter.

782 Figure 3 - Figure Supplement 5. Quality of 1H-fMRS spectra. Averaged spectra across

783 individual epochs (Left: REST; Center: PF; Right: UF) of the subset of subjects (N=16) who had

784 a reliable quantification for both Lac and Glu.

785 Figure $\quad 3$-Source Data 1. Demographics and 1H-fMRS study parameters.

786 Figure $\quad 3$ - Source Data 2. Modulations of metabolic profile of V1 during PF and UF 787 stimulations.

788 Figure $\quad 3$ - Source Data 3. Datasets and Matlab scripts for generating panels from Figure $789 \quad 3$ and associated figure supplements. 
Aalling NN, Nedergaard M, DiNuzzo M. 2018. Cerebral Metabolic Changes During Sleep. Current neurology and neuroscience reports 18(9):57. Alais D, Locke SM, Leung J, Van der Burg E. 2016. No attentional capture from invisible flicker. Scientific Reports 6(1):29296.

Askew CE, Lopez AJ, Wood MA, Metherate R. 2019. Nicotine excites VIP interneurons to disinhibit pyramidal neurons in auditory cortex. Synapse (New York, NY) 73(9):e22116.

Bednarik P, Tkac I, Giove F, DiNuzzo M, Deelchand DK, Emir UE, Eberly LE, Mangia S. 2015. Neurochemical and BOLD responses during neuronal activation measured in the human visual cortex at 7 Tesla. J Cereb Blood Flow Metab 35(4):601-610.

Bednarik P, Tkac I, Giove F, Eberly LE, Deelchand DK, Barreto FR, Mangia S. 2018. Neurochemical responses to chromatic and achromatic stimuli in the human visual cortex. J Cereb Blood Flow Metab 38(2):347-359.

Boillat Y, Xin L, van der Zwaag W, Gruetter R. 2020. Metabolite concentration changes associated with positive and negative BOLD responses in the human visual cortex: A functional MRS study at 7 Tesla. J Cereb Blood Flow Metab 40(3):488-500.

Boly M, Faymonville ME, Peigneux P, Lambermont B, Damas P, Del Fiore G, Degueldre C, Franck G, Luxen A, Lamy M, Moonen G, Maquet P, Laureys S. 2004. Auditory processing in severely brain injured patients: differences between the minimally conscious state and the persistent vegetative state. Arch Neurol 61(2):233-238.

Bueschke N, Amaral-Silva LD, Hu M, Santin JM. 2021. Lactate ions induce synaptic plasticity to enhance output from the central respiratory network. J Physiol.

Buxton RB, Griffeth VE, Simon AB, Moradi F, Shmuel A. 2014. Variability of the coupling of blood flow and oxygen metabolism responses in the brain: a problem for interpreting BOLD studies but potentially a new window on the underlying neural activity. Frontiers in neuroscience 8:139.

Chai Y, Handwerker DA, Marrett S, Gonzalez-Castillo J, Merriam EP, Hall A, Molfese PJ, Bandettini PA. 2019. Visual temporal frequency preference shows a distinct cortical architecture using fMRI. Neuroimage 197:13-23.

Chen Z, Silva AC, Yang J, Shen J. 2005. Elevated endogenous GABA level correlates with decreased fMRI signals in the rat brain during acute inhibition of GABA transaminase. J Neurosci Res 79(3):383-391.

Cox RW. 1996. AFNI: software for analysis and visualization of functional magnetic resonance neuroimages.

Computers and biomedical research, an international journal 29(3):162-173.

Crick F, Koch C. 1995. Are we aware of neural activity in primary visual cortex? Nature 375(6527):121-123. D'Souza RD, Burkhalter A. 2017. A Laminar Organization for Selective Cortico-Cortical Communication. Frontiers in neuroanatomy 11:71-71.

de Graaf RA, Brown PB, Mclntyre S, Nixon TW, Behar KL, Rothman DL. 2006. High magnetic field water and metabolite proton T1 and T2 relaxation in rat brain in vivo. Magn Reson Med 56(2):386-394.

de Graaf RA. 2007. In Vivo NMR Spectroscopy: Wiley \& Sons.

Descalzi G, Gao V, Steinman MQ, Suzuki A, Alberini CM. 2019. Lactate from astrocytes fuels learning-induced mRNA translation in excitatory and inhibitory neurons. Communications Biology 2(1):247.

Diaz-Garcia CM, Mongeon R, Lahmann C, Koveal D, Zucker H, Yellen G. 2017. Neuronal Stimulation Triggers

Neuronal Glycolysis and Not Lactate Uptake. Cell Metab 26(2):361-374.e364.

Dienel GA. 2019a. Brain Glucose Metabolism: Integration of Energetics with Function. Physiol Rev 99(1):949-1045.

Dienel GA. 2019b. Does shuttling of glycogen-derived lactate from astrocytes to neurons take place during neurotransmission and memory consolidation? J Neurosci Res.

DiNuzzo M. 2016. Astrocyte-Neuron Interactions during Learning May Occur by Lactate Signaling Rather than Metabolism. Front Integr Neurosci 10:2.

DiNuzzo M, Giove F. 2012. Activity-dependent energy budget for neocortical signaling: effect of short-term synaptic plasticity on the energy expended by spiking and synaptic activity. J Neurosci Res 90(11):2094-2102.

DiNuzzo M, Mangia S, Maraviglia B, Giove F. 2014. Effect of short-term synaptic plasticity on the relationship between neuronal activity, BOLD, CMRO2 and CMRGIc studied by metabolic modeling of neuron-glia interaction. Proc Intl Soc Magn Reson Med 22:1884.

DiNuzzo M, Maraviglia B, Giove F. 2011. Why does the brain (not) have glycogen? Bioessays 33(5):319-326. DiNuzzo M, Mascali D, Moraschi M, Bussu G, Maugeri L, Mangini F, Fratini M, Giove F. 2019. Brain Networks Underlying Eye's Pupil Dynamics. Frontiers in neuroscience 13:965.

DiNuzzo M, Nedergaard M. 2017. Brain energetics during the sleep-wake cycle. Current opinion in neurobiology 47:65-72.

Donahue MJ, Rane S, Hussey E, Mason E, Pradhan S, Waddell KW, Ally BA. 2014. y-Aminobutyric acid (GABA) concentration inversely correlates with basal perfusion in human occipital lobe. J Cereb Blood Flow Metab 34(3):532541. 
Fernandes CC, Lanz B, Chen C, Morris PG. 2020. Measurement of brain lactate during visual stimulation using a long TE semi-LASER sequence at 7 T. NMR in biomedicine 33(4):e4223.

Fox PT, Mintun MA, Raichle ME, Miezin FM, Allman JM, Van Essen DC. 1986. Mapping human visual cortex with positron emission tomography. Nature 323(6091):806-809.

Gail A, Brinksmeyer HJ, Eckhorn R. 2004. Perception-related modulations of local field potential power and

coherence in primary visual cortex of awake monkey during binocular rivalry. Cereb Cortex 14(3):300-313.

Gasselin C, Hohl B, Vernet A, Crochet S, Petersen CCH. 2021. Cell-type-specific nicotinic input disinhibits mouse barrel cortex during active sensing. Neuron 109(5):778-787.e773.

Gelbard-Sagiv H, Magidov E, Sharon H, Hendler T, Nir Y. 2018. Noradrenaline Modulates Visual Perception and Late Visually Evoked Activity. Curr Biol 28(14):2239-2249.e2236.

Gruetter R, Tkác I. 2000. Field mapping without reference scan using asymmetric echo-planar techniques. Magn Reson Med 43(2):319-323.

Gur M, Snodderly DM. 1997. A dissociation between brain activity and perception: chromatically opponent cortical neurons signal chromatic flicker that is not perceived. Vision Res 37(4):377-382.

Harris RA, Lone A, Lim H, Martinez F, Frame AK, Scholl TJ, Cumming RC. 2019. Aerobic Glycolysis Is Required for Spatial Memory Acquisition But Not Memory Retrieval in Mice. eNeuro 6(1).

Hecht S, Shlaer S. 1936. INTERMITTENT STIMULATION BY LIGHT : V. THE RELATION BETWEEN INTENSITY AND CRITICAL FREQUENCY FOR DIFFERENT PARTS OF THE SPECTRUM. J Gen Physiol 19(6):965-977. Herculano-Houzel S. 2011. Scaling of brain metabolism with a fixed energy budget per neuron: implications for neuronal activity, plasticity and evolution. PLoS One 6(3):e17514.

Herrera-López G, Griego E, Galván EJ. 2020. Lactate induces synapse-specific potentiation on CA3 pyramidal cells of rat hippocampus. PLoS One 15(11):e0242309.

Herrmann CS. 2001. Human EEG responses to 1-100 Hz flicker: resonance phenomena in visual cortex and their potential correlation to cognitive phenomena. Exp Brain Res 137(3-4):346-353.

Hertz L, Chen Y. 2017. Integration between Glycolysis and Glutamate-Glutamine Cycle Flux May Explain Preferential Glycolytic Increase during Brain Activation, Requiring Glutamate. Front Integr Neurosci 11(18):doi:

10.3389/fnint.2017.00018.

Hertz L, Rothman DL. 2017. Glutamine-glutamate cycle flux is similar in cultured astrocytes and brain and both glutamate production and oxidation are mainly catalyzed by aspartate aminotransferase. Biology (Basel) 6(1). Hesselmann G, Malach R. 2011. The link between fMRI-BOLD activation and perceptual awareness is "streaminvariant" in the human visual system. Cereb Cortex 21(12):2829-2837.

Ip IB, Berrington A, Hess AT, Parker AJ, Emir UE, Bridge H. 2017. Combined fMRI-MRS acquires simultaneous glutamate and BOLD-fMRI signals in the human brain. Neuroimage 155:113-119.

Jamadar SD, Ward PGD, Liang EX, Orchard ER, Chen Z, Egan GF. 2021. Metabolic and Hemodynamic RestingState Connectivity of the Human Brain: A High-Temporal Resolution Simultaneous BOLD-fMRI and FDG-fPET Multimodality Study. Cerebral Cortex.

Jenkinson M, Beckmann CF, Behrens TE, Woolrich MW, Smith SM. 2012. FSL. Neuroimage 62(2):782-790.

Jiang Y, Zhou K, He S. 2007. Human visual cortex responds to invisible chromatic flicker. Nat Neurosci 10(5):657662.

Jourdain P, Rothenfusser K, Ben-Adiba C, Allaman I, Marquet P, Magistretti PJ. 2018. Dual action of L-Lactate on the activity of NR2B-containing NMDA receptors: from potentiation to neuroprotection. Sci Rep 8(1):13472.

Just N, Xin L, Frenkel H, Gruetter R. 2013. Characterization of sustained BOLD activation in the rat barrel cortex and neurochemical consequences. Neurolmage 74:343-351.

Kastner S, O'Connor DH, Fukui MM, Fehd HM, Herwig U, Pinsk MA. 2004. Functional imaging of the human lateral geniculate nucleus and pulvinar. J Neurophysiol 91(1):438-448.

Kauffmann L, Ramanoël S, Peyrin C. 2014. The neural bases of spatial frequency processing during scene perception. Frontiers in Integrative Neuroscience 8(37).

Klose U. 1990. In vivo proton spectroscopy in presence of eddy currents. Magn Reson Med 14(1):26-30.

Kobayashi R, Maruoka J, Norimoto H, Ikegaya Y, Kume K, Ohsawa M. 2019. Involvement of I-lactate in hippocampal dysfunction of type I diabetes. Journal of pharmacological sciences 141(1):79-82.

Kwong KK, Belliveau JW, Chesler DA, Goldberg IE, Weisskoff RM, Poncelet BP, Kennedy DN, Hoppel BE, Cohen MS, Turner R, et al. 1992. Dynamic magnetic resonance imaging of human brain activity during primary sensory stimulation. Proc Natl Acad Sci U S A 89(12):5675-5679.

Lamme VAF, Supèr H, Landman R, Roelfsema PR, Spekreijse H. 2000. The role of primary visual cortex (V1) in visual awareness. Vision Research 40(10):1507-1521.

Laureys S, Faymonville ME, Degueldre C, Fiore GD, Damas P, Lambermont B, Janssens N, Aerts J, Franck G, Luxen A, Moonen G, Lamy M, Maquet P. 2000. Auditory processing in the vegetative state. Brain 123 ( Pt 8):1589-1601. 
Laureys S, Faymonville ME, Peigneux P, Damas P, Lambermont B, Del Fiore G, Degueldre C, Aerts J, Luxen A, Franck G, Lamy M, Moonen G, Maquet P. 2002. Cortical processing of noxious somatosensory stimuli in the persistent vegetative state. Neuroimage 17(2):732-741. Lauritzen M. 2001. Relationship of spikes, synaptic activity, and local changes of cerebral blood flow. J Cereb Blood Flow Metab 21(12):1367-1383. Leopold DA, Logothetis NK. 1996. Activity changes in early visual cortex reflect monkeys' percepts during binocular rivalry. Nature 379(6565):549-553. consumption, and ATP production in human visual cortex. Proc Natl Acad Sci U S A 107(18):8446-8451. Lin Y, Stephenson MC, Xin L, Napolitano A, Morris PG. 2012. Investigating the metabolic changes due to visual stimulation using functional proton magnetic resonance spectroscopy at 7 T. J Cereb Blood Flow Metab 32(8):14841495.

Logothetis NK. 2008. What we can do and what we cannot do with fMRI. Nature 453(7197):869-878.

Logothetis NK, Pauls J, Augath M, Trinath T, Oeltermann A. 2001. Neurophysiological investigation of the basis of the fMRI signal. Nature 412(6843):150-157.

Lorenceau J. 1987. Recovery from contrast adaptation: effects of spatial and temporal frequency. Vision Res 27(12):2185-2191.

Lundquist AJ, Gallagher TJ, Petzinger GM, Jakowec MW. 2021. Exogenous I-lactate promotes astrocyte plasticity but is not sufficient for enhancing striatal synaptogenesis or motor behavior in mice. J Neurosci Res 99(5):1433-1447. Maier A, Wilke M, Aura C, Zhu C, Ye FQ, Leopold DA. 2008. Divergence of fMRI and neural signals in V1 during perceptual suppression in the awake monkey. Nat Neurosci 11(10):1193-1200.

Mangia S, Giove F, Dinuzzo M. 2012. Metabolic pathways and activity-dependent modulation of glutamate concentration in the human brain. Neurochem Res 37(11):2554-2561.

Mangia S, Giove F, Tkac I, Logothetis NK, Henry PG, Olman CA, Maraviglia B, Di Salle F, Ugurbil K. 2009. Metabolic and hemodynamic events after changes in neuronal activity: current hypotheses, theoretical predictions and in vivo NMR experimental findings. J Cereb Blood Flow Metab 29(3):441-463.

Mangia S, Tkac I, Gruetter R, Van de Moortele PF, Maraviglia B, Ugurbil K. 2007a. Sustained neuronal activation raises oxidative metabolism to a new steady-state level: evidence from $1 \mathrm{H}$ NMR spectroscopy in the human visual cortex. J Cereb Blood Flow Metab 27(5):1055-1063.

Mangia S, Tkac I, Logothetis NK, Gruetter R, Van de Moortele PF, Ugurbil K. 2007b. Dynamics of lactate concentration and blood oxygen level-dependent effect in the human visual cortex during repeated identical stimuli. $J$ Neurosci Res 85(15):3340-3346.

Margineanu MB, Mahmood H, Fiumelli H, Magistretti PJ. 2018. L-Lactate Regulates the Expression of Synaptic Plasticity and Neuroprotection Genes in Cortical Neurons: A Transcriptome Analysis. Front Mol Neurosci 11:375. Min BK, Kim HS, Pinotsis DA, Pantazis D. 2020. Thalamocortical inhibitory dynamics support conscious perception. Neuroimage 220:117066.

Moradi F, Buračas GT, Buxton RB. 2012. Attention strongly increases oxygen metabolic response to stimulus in primary visual cortex. Neuroimage 59(1):601-607.

Muthukumaraswamy SD, Edden RA, Jones DK, Swettenham JB, Singh KD. 2009. Resting GABA concentration predicts peak gamma frequency and fMRI amplitude in response to visual stimulation in humans. Proc Natl Acad Sci U S A 106(20):8356-8361.

Naressi A, Couturier C, Devos JM, Janssen M, Mangeat C, de Beer R, Graveron-Demilly D. 2001. Java-based graphical user interface for the MRUI quantitation package. Magma (New York, NY) 12(2-3):141-152.

Naylor E, Aillon DV, Barrett BS, Wilson GS, Johnson DA, Johnson DA, Harmon HP, Gabbert S, Petillo PA. 2012. Lactate as a biomarker for sleep. Sleep 35(9):1209-1222.

Naylor E, Aillon DV, Gabbert S, Harmon H, Johnson DA, Wilson GS, Petillo PA. 2011. Simultaneous real-time measurement of EEG/EMG and L-glutamate in mice: A biosensor study of neuronal activity during sleep. Journal of electroanalytical chemistry (Lausanne, Switzerland) 656(1-2):106-113.

Newman LA, Korol DL, Gold PE. 2011. Lactate produced by glycogenolysis in astrocytes regulates memory processing. PLoS One 6(12):e28427.

Niven JE, Laughlin SB. 2008. Energy limitation as a selective pressure on the evolution of sensory systems. The Journal of experimental biology 211(Pt 11):1792-1804.

Noack R, Manjesh C, Ruszinko M, Siegelmann H, Kozma R. Resting state neural networks and energy metabolism; 2017 14-19 May 2017. p 228-235.

Norcia AM, Appelbaum LG, Ales JM, Cottereau BR, Rossion B. 2015. The steady-state visual evoked potential in vision research: A review. Journal of vision 15(6):4.

Northoff G, Walter M, Schulte RF, Beck J, Dydak U, Henning A, Boeker H, Grimm S, Boesiger P. 2007. GABA concentrations in the human anterior cingulate cortex predict negative BOLD responses in fMRI. Nat Neurosci 10(12):1515-1517. 
Pastor MA, Artieda J, Arbizu J, Valencia M, Masdeu JC. 2003. Human cerebral activation during steady-state visualevoked responses. J Neurosci 23(37):11621-11627.

Peca S, Carnì M, Di Bonaventura C, Aprile T, Hagberg GE, Giallonardo AT, Manfredi M, Mangia S, Garreffa G, Maraviglia B, Giove F. 2010. Metabolic correlatives of brain activity in a FOS epilepsy patient. NMR in biomedicine 23(2):170-178.

Pi HJ, Hangya B, Kvitsiani D, Sanders JI, Huang ZJ, Kepecs A. 2013. Cortical interneurons that specialize in disinhibitory control. Nature 503(7477):521-524.

Polonsky A, Blake R, Braun J, Heeger DJ. 2000. Neuronal activity in human primary visual cortex correlates with perception during binocular rivalry. Nat Neurosci 3(11):1153-1159.

Power JD, Barnes KA, Snyder AZ, Schlaggar BL, Petersen SE. 2012. Spurious but systematic correlations in functional connectivity MRI networks arise from subject motion. Neurolmage 59(3):2142-2154.

Provencher SW. 1993. Estimation of metabolite concentrations from localized in vivo proton NMR spectra. Magn Reson Med 30(6):672-679.

Regan D. 1989. Human brain electrophysiology: evoked potentials and evoked magnetic fields in science and medicine. New York: Elsevier.

Sachidhanandam S, Sreenivasan V, Kyriakatos A, Kremer Y, Petersen CC. 2013. Membrane potential correlates of sensory perception in mouse barrel cortex. Nat Neurosci 16(11):1671-1677.

Salminen-Vaparanta N, Koivisto M, Vorobyev V, Alakurtti K, Revonsuo A. 2019. Does TMS on V3 block conscious visual perception? Neuropsychologia 128:223-231.

Scavuzzo CJ, Rakotovao I, Dickson CT. 2020. Differential effects of L- and D-lactate on memory encoding and consolidation: Potential role of HCAR1 signaling. Neurobiology of learning and memory 168:107151.

Schaller B, Mekle R, Xin L, Kunz N, Gruetter R. 2013. Net increase of lactate and glutamate concentration in activated human visual cortex detected with magnetic resonance spectroscopy at 7 tesla. J Neurosci Res 91(8):10761083.

Schaller B, Xin L, O'Brien K, Magill AW, Gruetter R. 2014. Are glutamate and lactate increases ubiquitous to physiological activation? A (1)H functional MR spectroscopy study during motor activation in human brain at 7Tesla. Neuroimage 93 Pt 1:138-145.

Shady S, MacLeod DIA, Fisher HS. 2004. Adaptation from invisible flicker. Proc Natl Acad Sci U S A 101(14):5170-5173.

Siesjo DP. 1978. Brain Energy Metabolism. New York: Wiley.

Singh KD, Smith AT, Greenlee MW. 2000. Spatiotemporal frequency and direction sensitivities of human visual areas measured using fMRI. Neuroimage 12(5):550-564.

Soher BJ, Young K, Bernstein A, Aygula Z, Maudsley AA. 2007. GAVA: spectral simulation for in vivo MRS applications. Journal of magnetic resonance (San Diego, Calif : 1997) 185(2):291-299.

Spetsieris PG, Ko JH, Tang CC, Nazem A, Sako W, Peng S, Ma Y, Dhawan V, Eidelberg D. 2015. Metabolic restingstate brain networks in health and disease. Proc Natl Acad Sci U S A 112(8):2563-2568.

Steinman MQ, Gao V, Alberini CM. 2016. The Role of Lactate-Mediated Metabolic Coupling between Astrocytes and Neurons in Long-Term Memory Formation. Frontiers in integrative neuroscience 10:10-10.

Su H, Zuo C, Zhang H, Jiao F, Zhang B, Tang W, Geng D, Guan Y, Shi S. 2018. Regional cerebral metabolism alterations affect resting-state functional connectivity in major depressive disorder. Quantitative Imaging in Medicine and Surgery 8(9):910-924.

Suzuki A, Stern SA, Bozdagi O, Huntley GW, Walker RH, Magistretti PJ, Alberini CM. 2011. Astrocyte-neuron lactate transport is required for long-term memory formation. Cell 144(5):810-823.

Thomas CG, Menon RS. 1998. Amplitude response and stimulus presentation frequency response of human primary visual cortex using BOLD EPI at 4 T. Magn Reson Med 40(2):203-209.

Thompson GJ. 2018. Neural and metabolic basis of dynamic resting state fMRI. Neuroimage 180(Pt B):448-462. Tkác I, Andersen P, Adriany G, Merkle H, Ugurbil K, Gruetter R. 2001. In vivo 1H NMR spectroscopy of the human brain at 7 T. Magn Reson Med 46(3):451-456.

Tkác I, Starcuk Z, Choi IY, Gruetter R. 1999. In vivo 1H NMR spectroscopy of rat brain at 1 ms echo time. Magn Reson Med 41(4):649-656.

Tong F. 2003. Primary visual cortex and visual awareness. Nat Rev Neurosci 4(3):219-229.

Vialatte FB, Maurice M, Dauwels J, Cichocki A. 2010. Steady-state visually evoked potentials: focus on essential paradigms and future perspectives. Prog Neurobiol 90(4):418-438.

Viswanathan A, Freeman RD. 2007. Neurometabolic coupling in cerebral cortex reflects synaptic more than spiking activity. Nat Neurosci 10(10):1308--1312.

Wang Q, Hu Y, Wan J, Dong B, Sun J. 2019. Lactate: A Novel Signaling Molecule in Synaptic Plasticity and Drug Addiction. Bioessays 41(8):e1900008.

Watanabe M, Cheng K, Murayama Y, Ueno K, Asamizuya T, Tanaka K, Logothetis N. 2011. Attention but not awareness modulates the BOLD signal in the human V1 during binocular suppression. Science 334(6057):829-831. 
Watkins S, Shams L, Tanaka S, Haynes JD, Rees G. 2006. Sound alters activity in human V1 in association with illusory visual perception. Neuroimage 31(3):1247-1256. Williams LE, Holtmaat A. 2019. Higher-Order Thalamocortical Inputs Gate Synaptic Long-Term Potentiation via Disinhibition. Neuron 101(1):91-102.e104.

1028

1029

1030

1031

1032

1033

1034

1035

1036

1037

1038

1039

1040

Wunderlich K, Schneider KA, Kastner S. 2005. Neural correlates of binocular rivalry in the human lateral geniculate nucleus. Nat Neurosci 8(11):1595-1602.

Yang J, Ruchti E, Petit JM, Jourdain P, Grenningloh G, Allaman I, Magistretti PJ. 2014. Lactate promotes plasticity gene expression by potentiating NMDA signaling in neurons. Proc Natl Acad Sci U S A 111(33):12228-12233.

Yu J, Hu H, Agmon A, Svoboda K. 2019. Recruitment of GABAergic Interneurons in the Barrel Cortex during Active Tactile Behavior. Neuron 104(2):412-427.e414.

Yuval-Greenberg S, Heeger DJ. 2013. Continuous flash suppression modulates cortical activity in early visual cortex. J Neurosci 33(23):9635-9643.

Zhu XH, Chen W. 2001. Observed BOLD effects on cerebral metabolite resonances in human visual cortex during visual stimulation: a functional (1)H MRS study at 4 T. Magn Reson Med 46(5):841-847.

Zuend M, Saab AS, Wyss MT, Ferrari KD, Hösli L, Looser ZJ, Stobart JL, Duran J, Guinovart JJ, Barros LF, Weber B. 2020. Arousal-induced cortical activity triggers lactate release from astrocytes. Nat Metab 2(2):179-191.

1041

1042

1043 






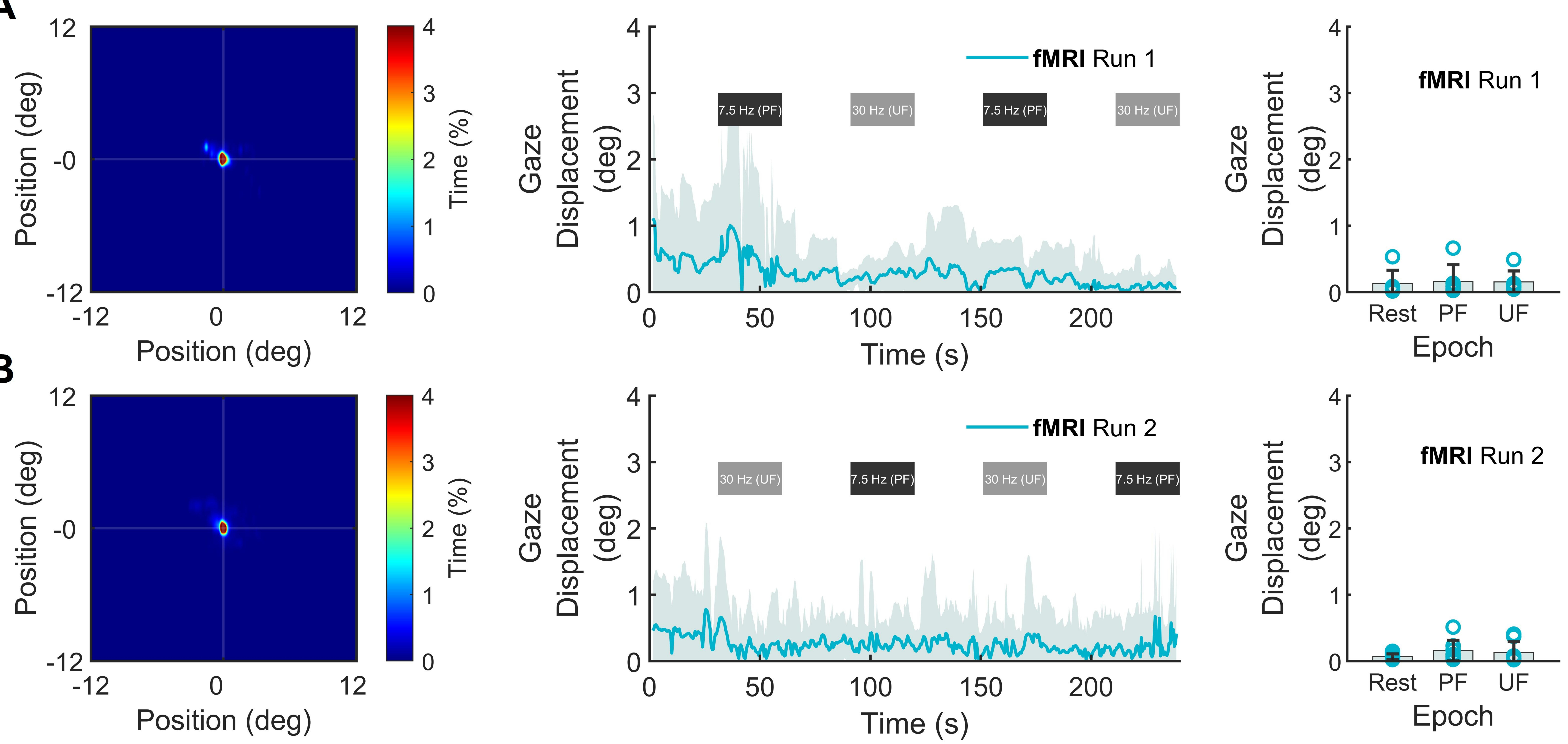


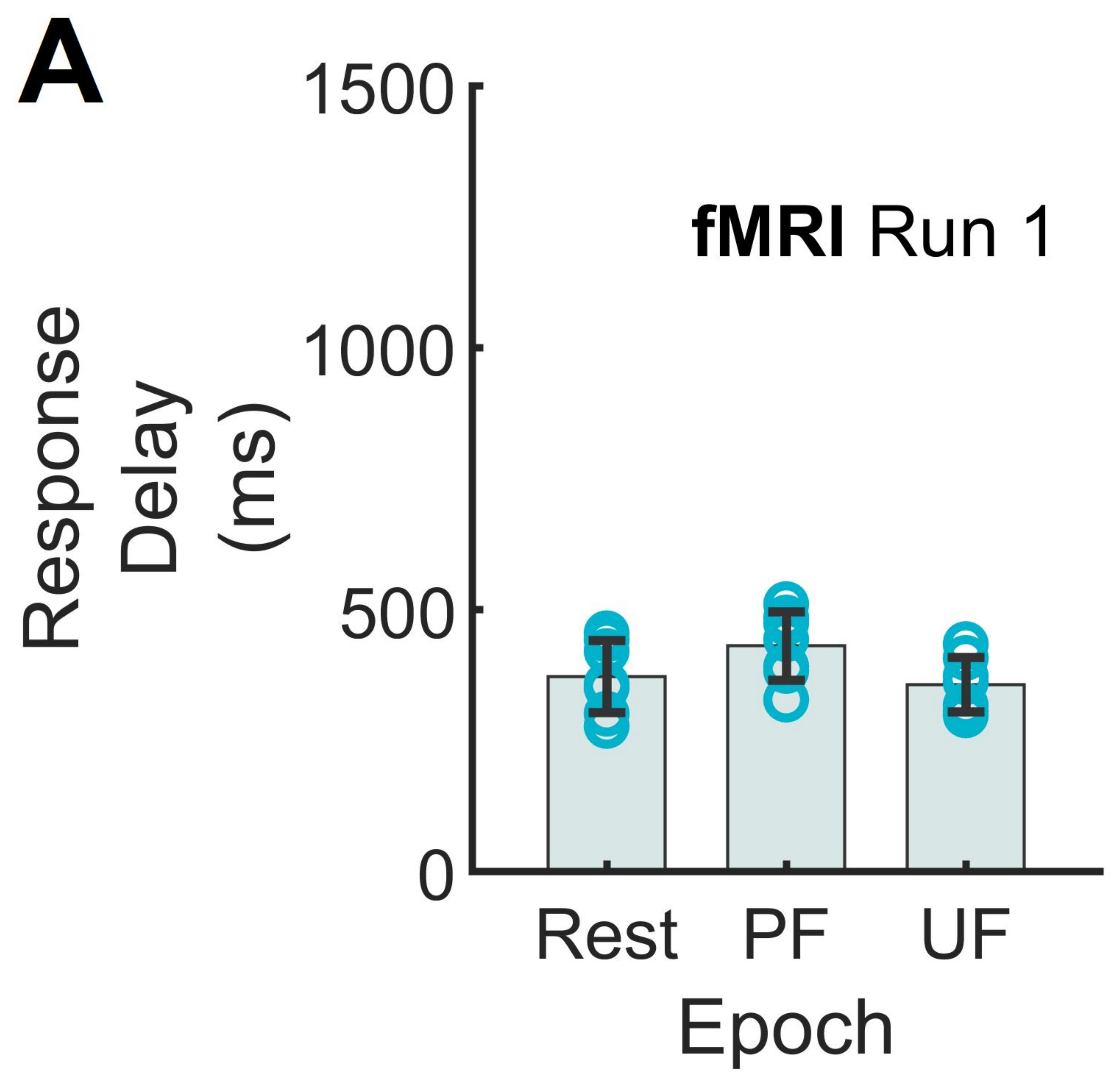

B

1500

fMRI Run 2

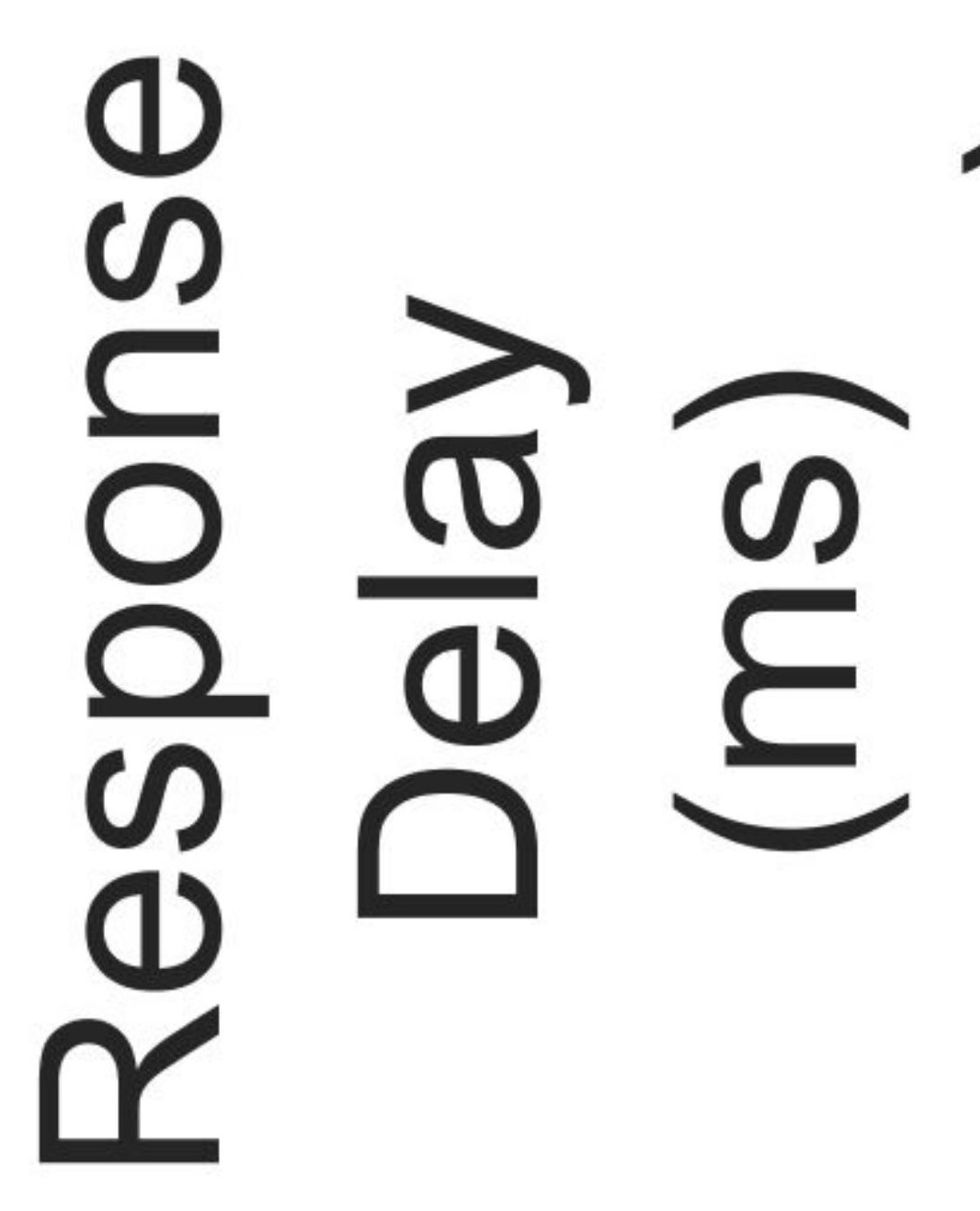

0

1000

o

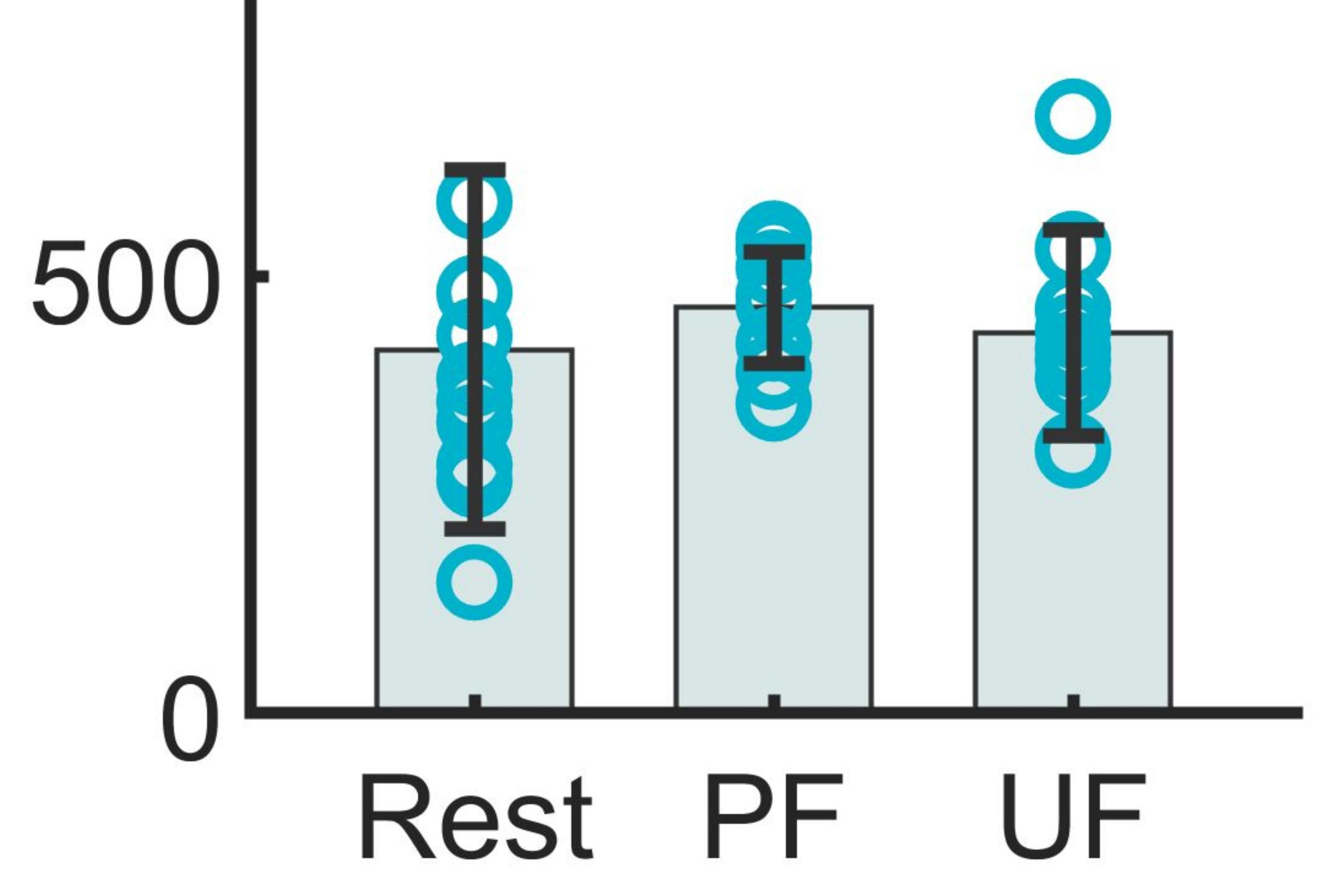

Epoch 


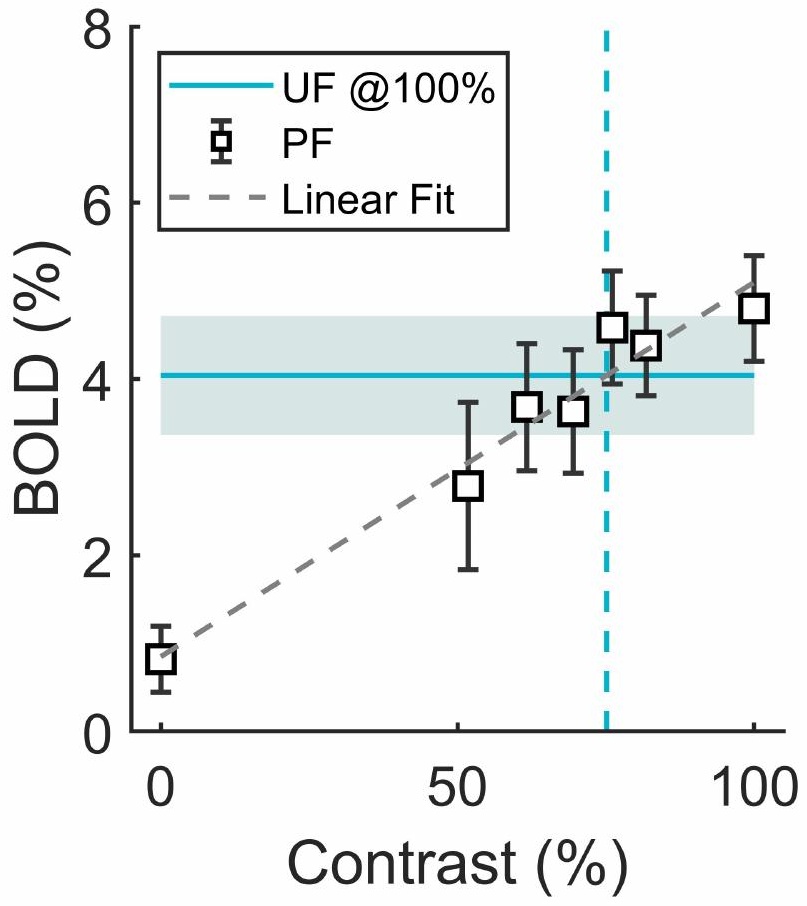


A


C

PF, UF, Overlap
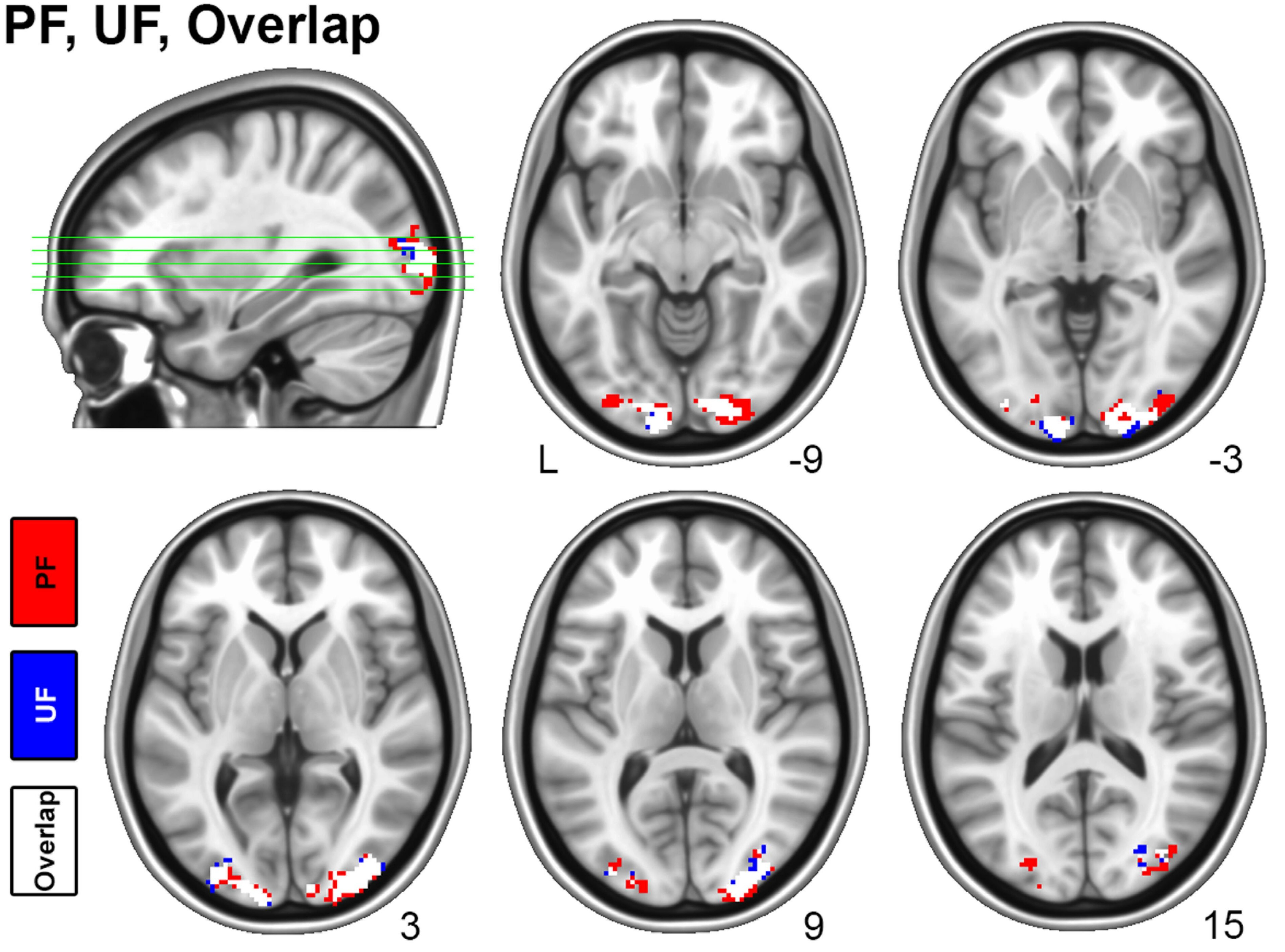


D

$$
\text { PF }(7.5 \mathrm{~Hz})>\text { UF }(30 \mathrm{~Hz})
$$
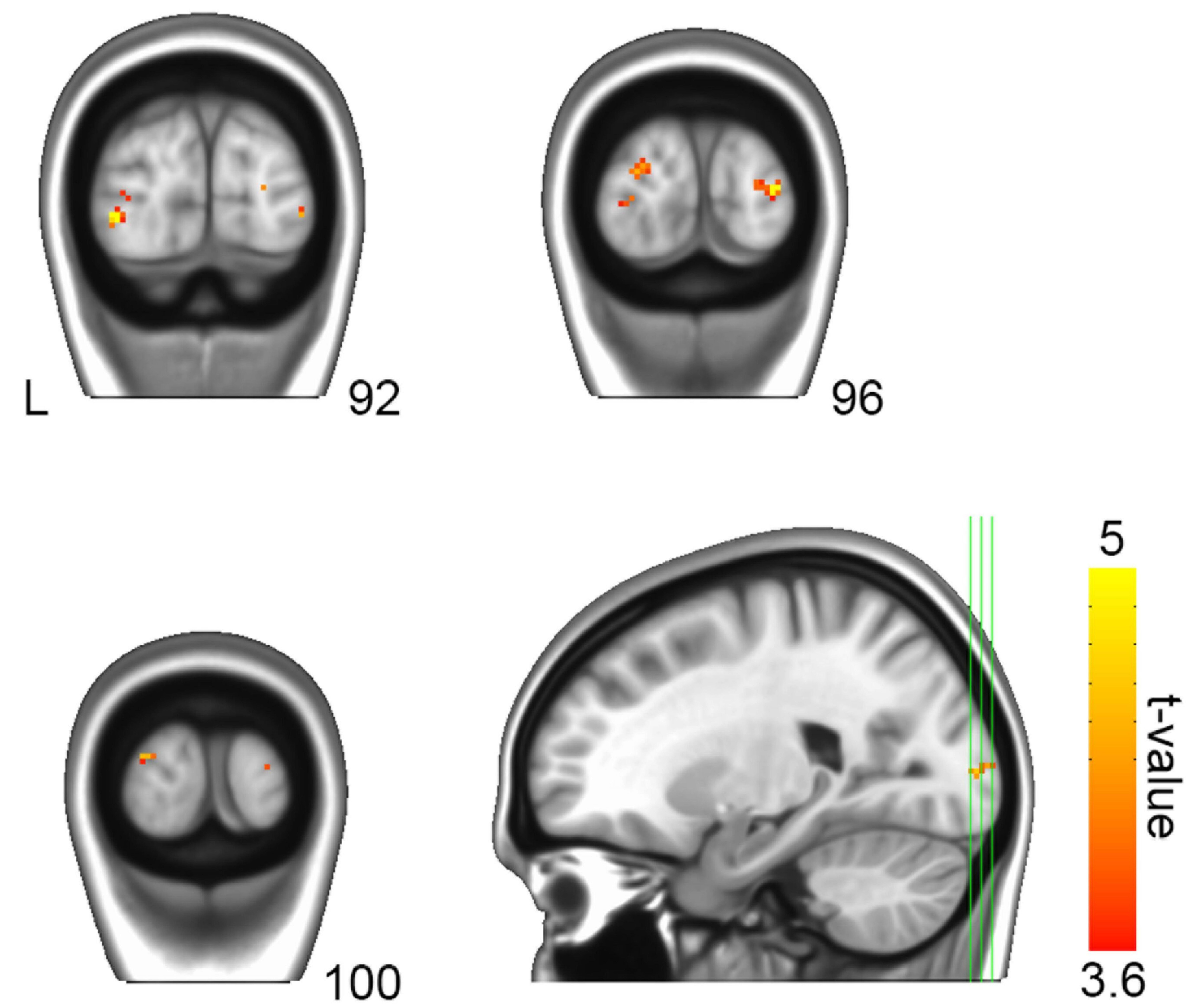
Intensity (\% Peak)

1H-fMRS VOI
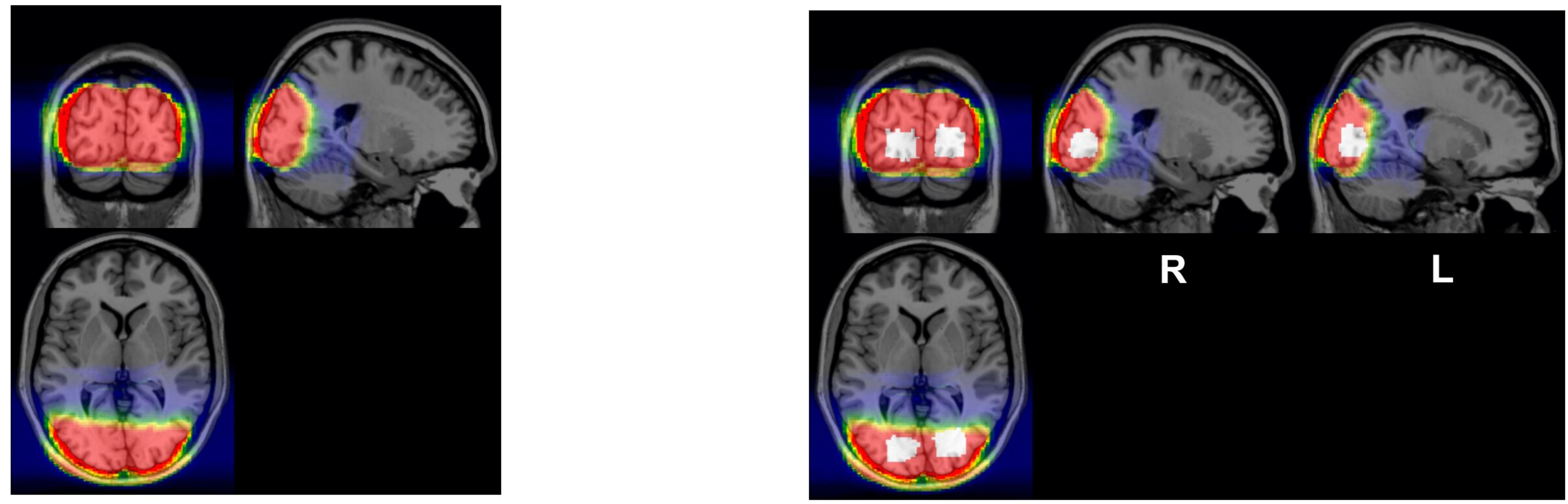

BA17

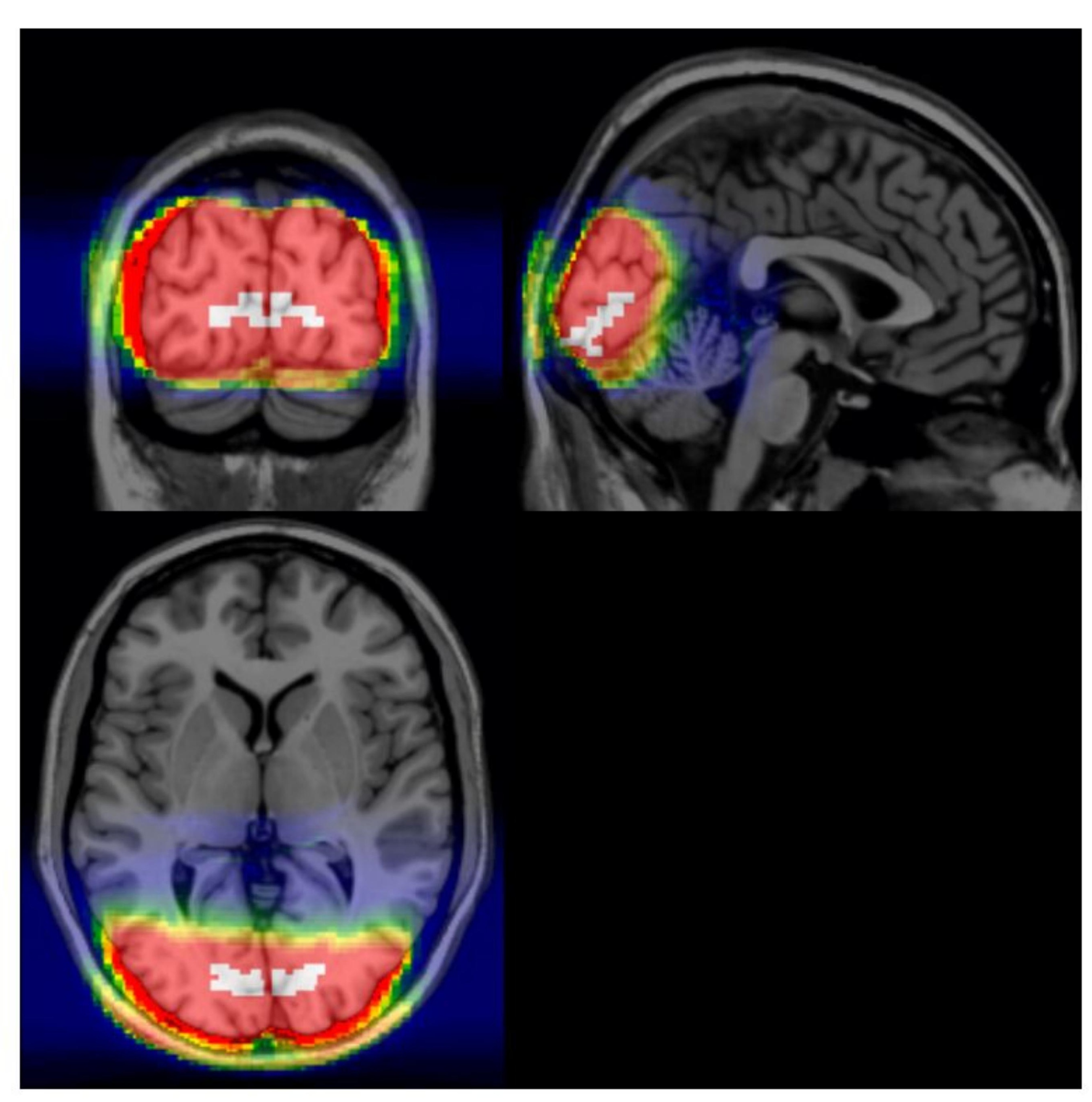

BA18

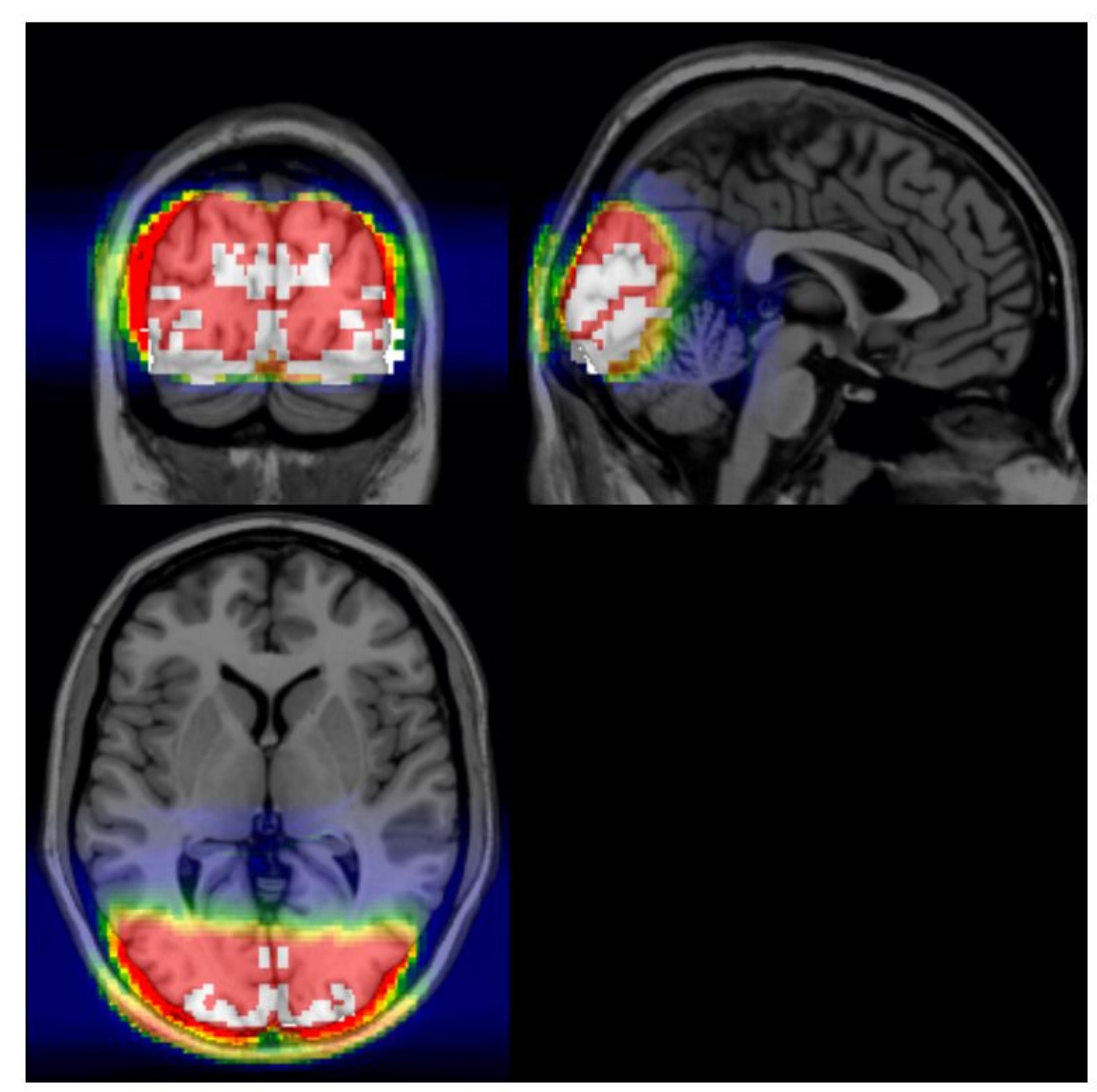

BA19

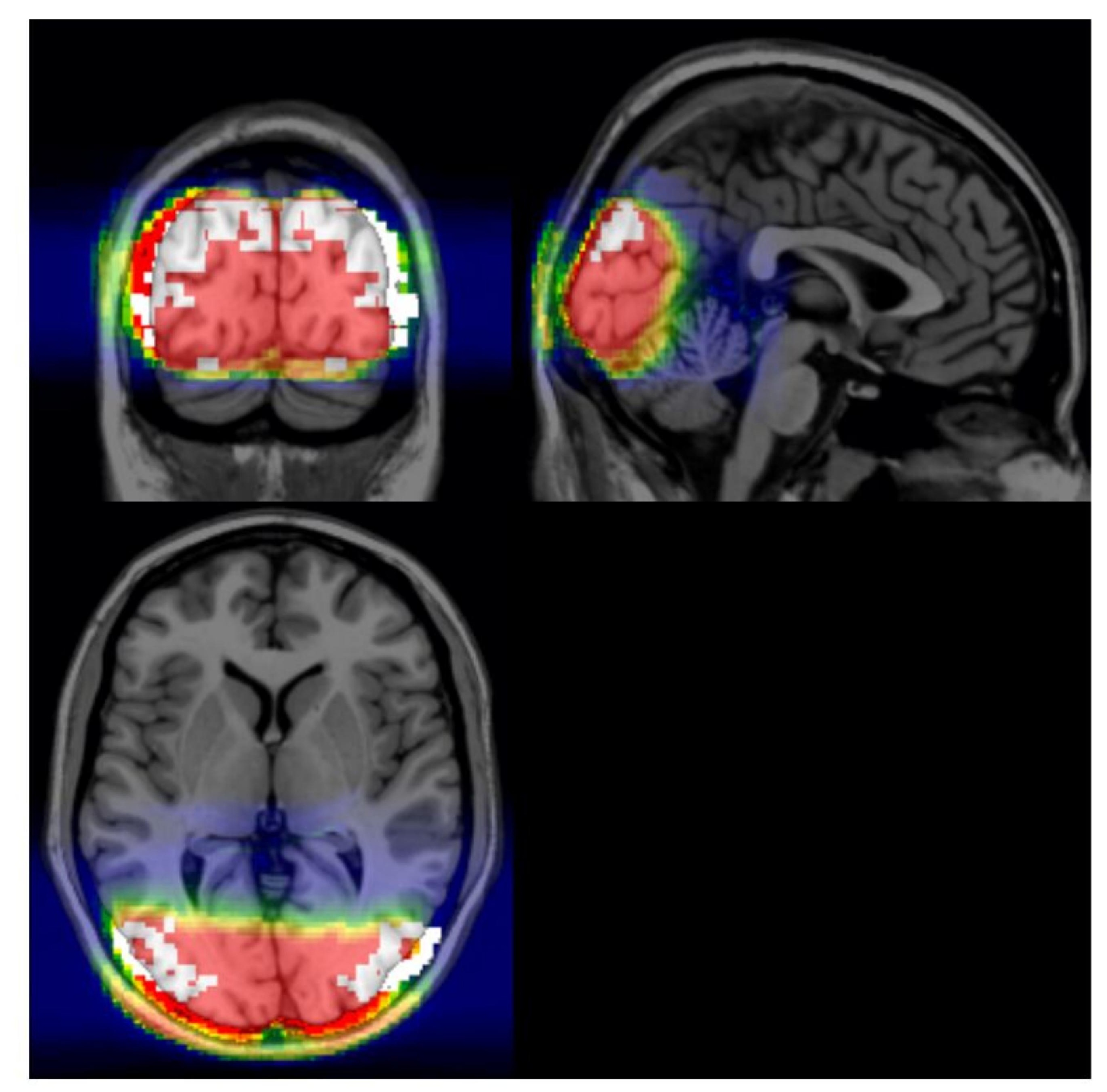

\section{B}

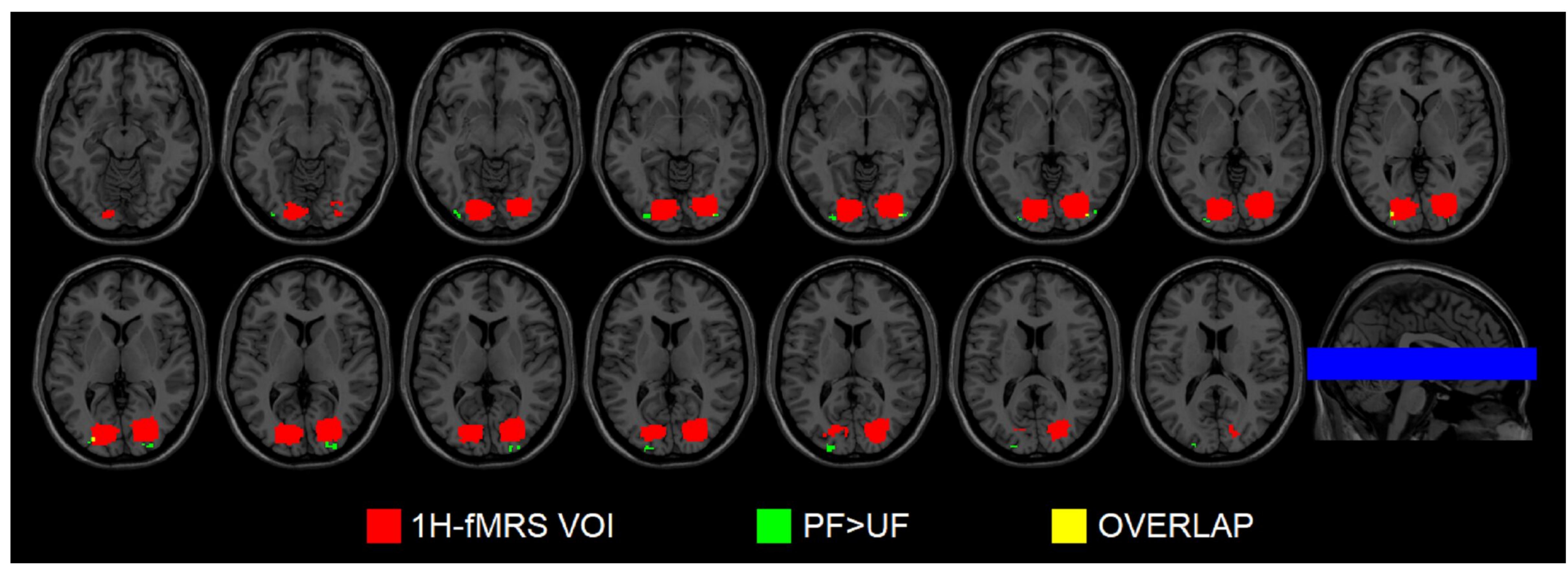


A

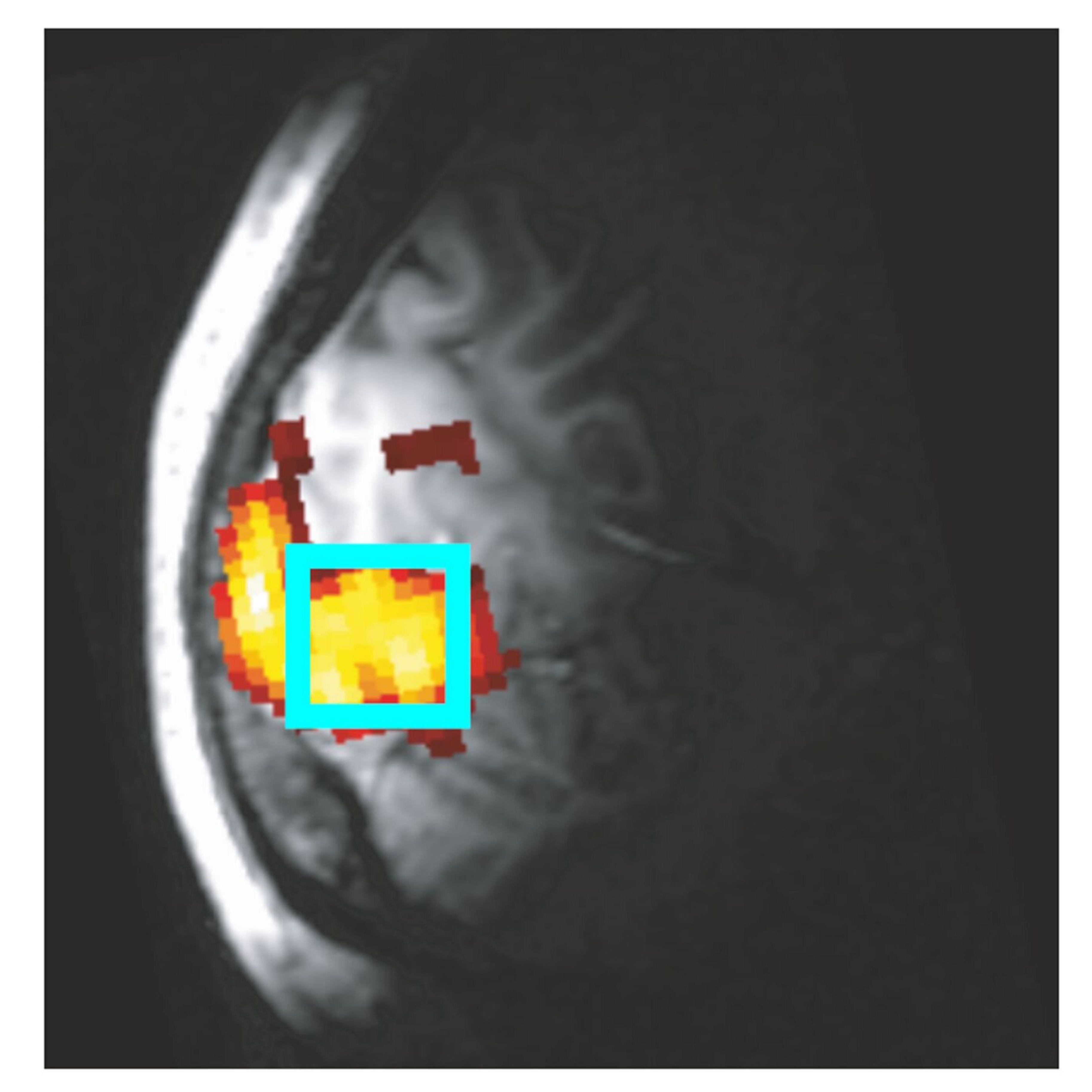

tNAA

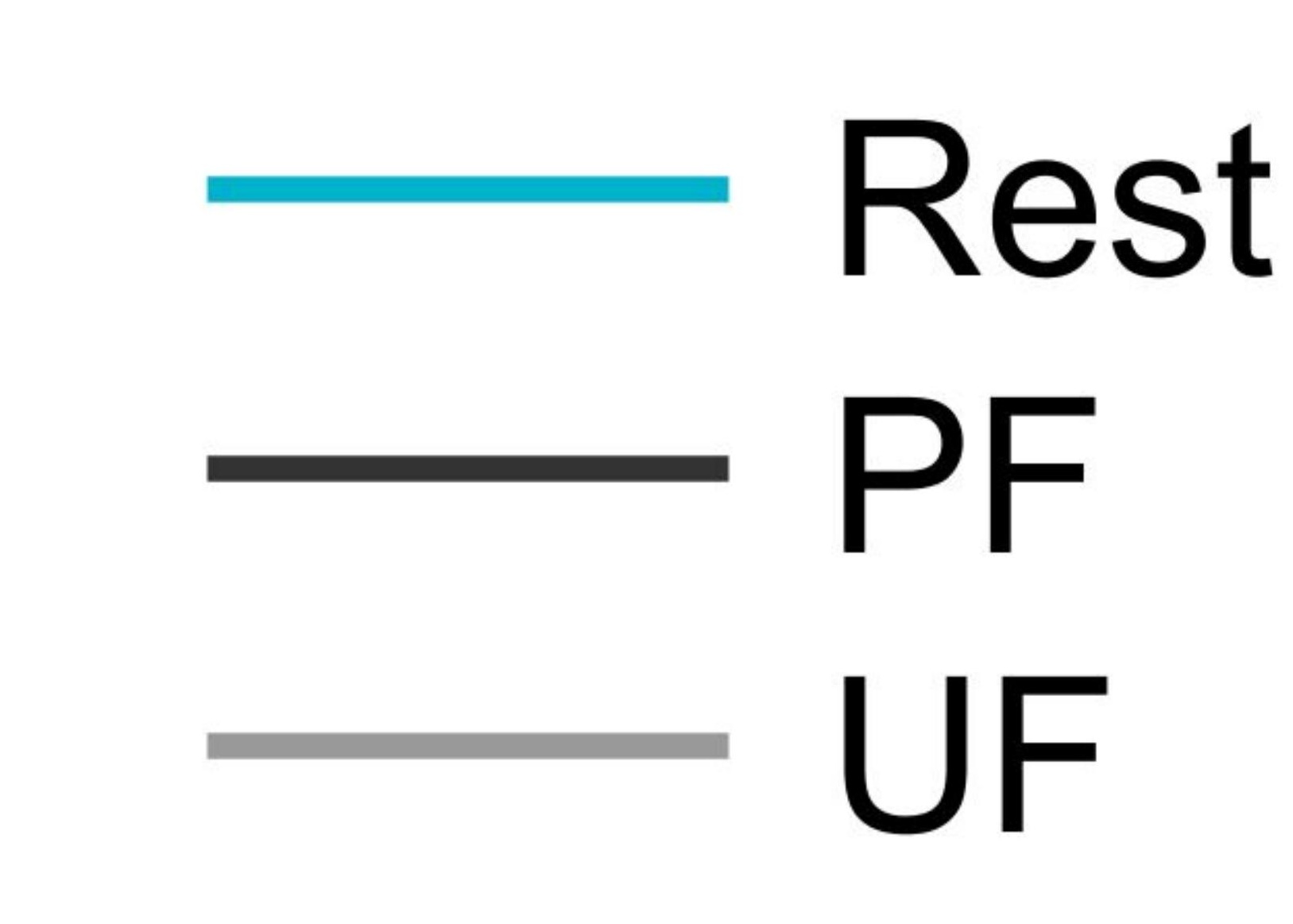

$\mathrm{tCr}$

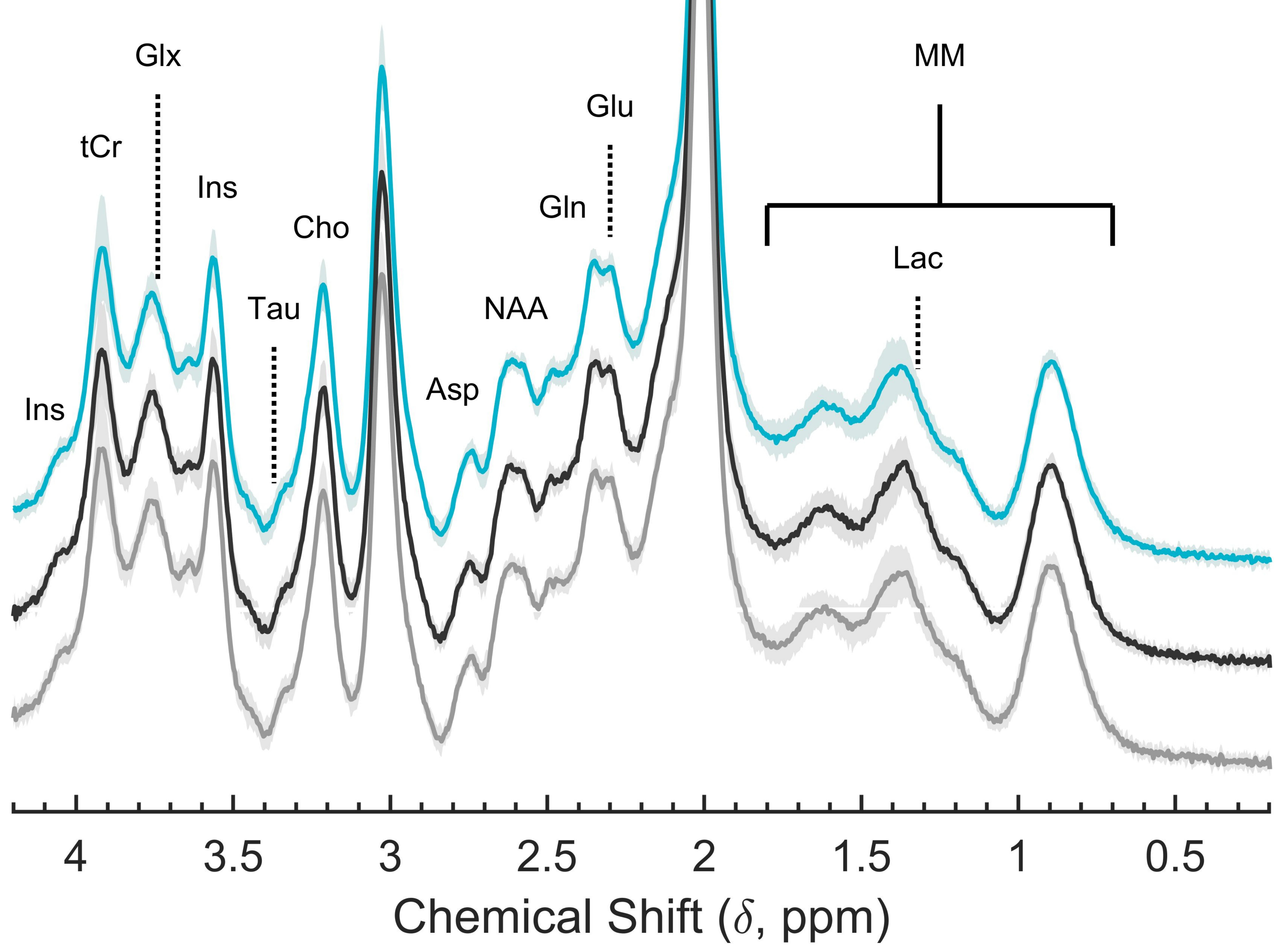

C

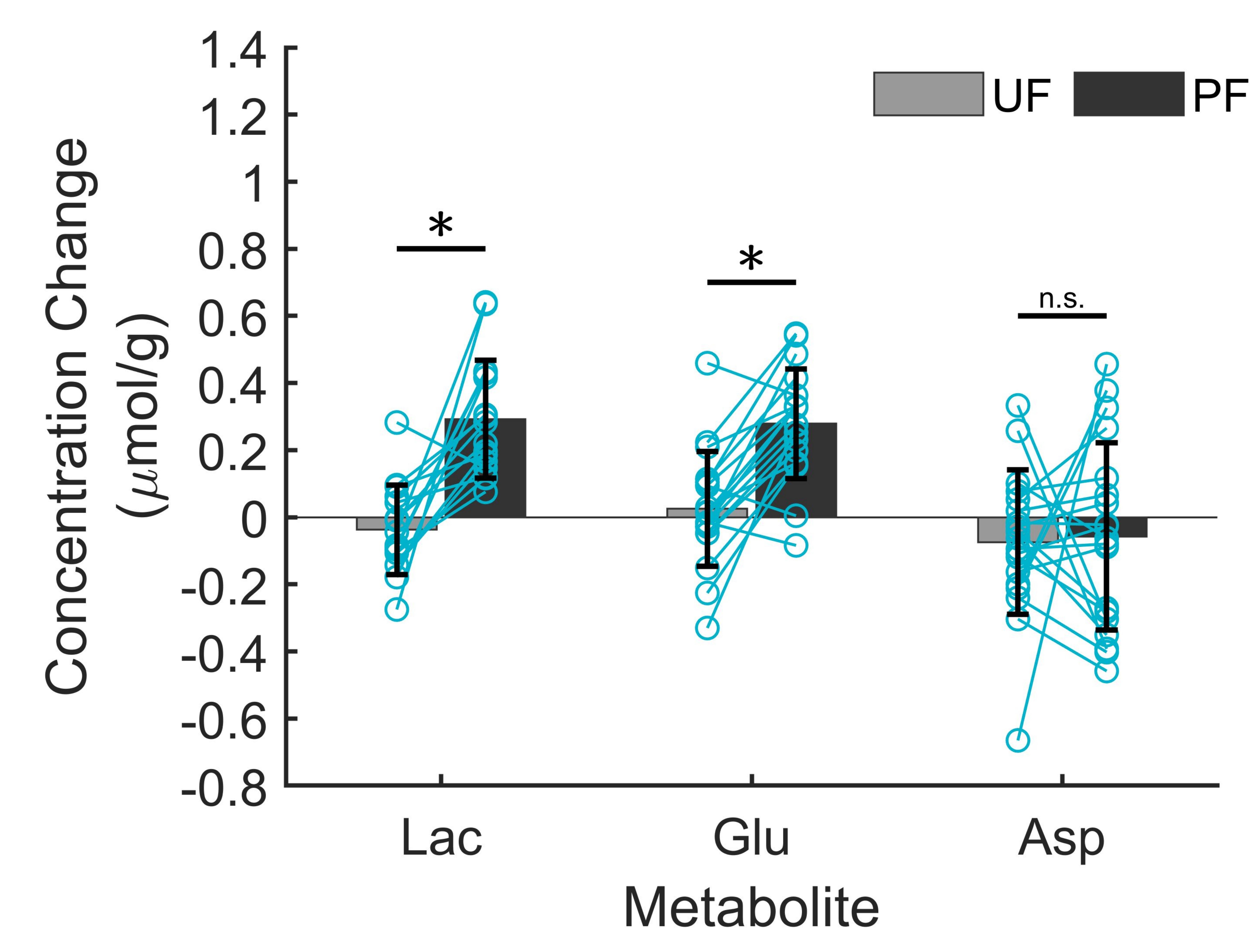

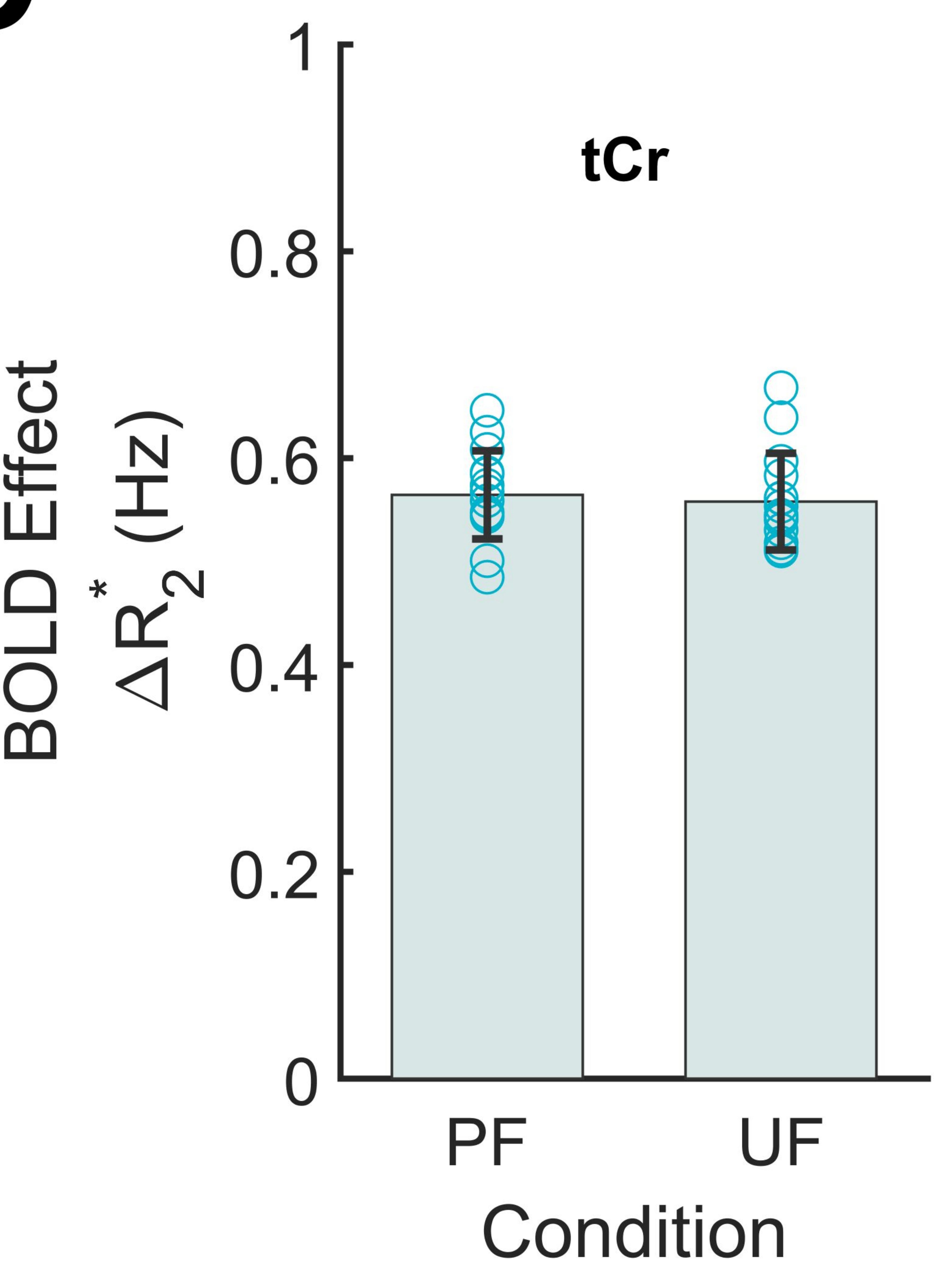

D

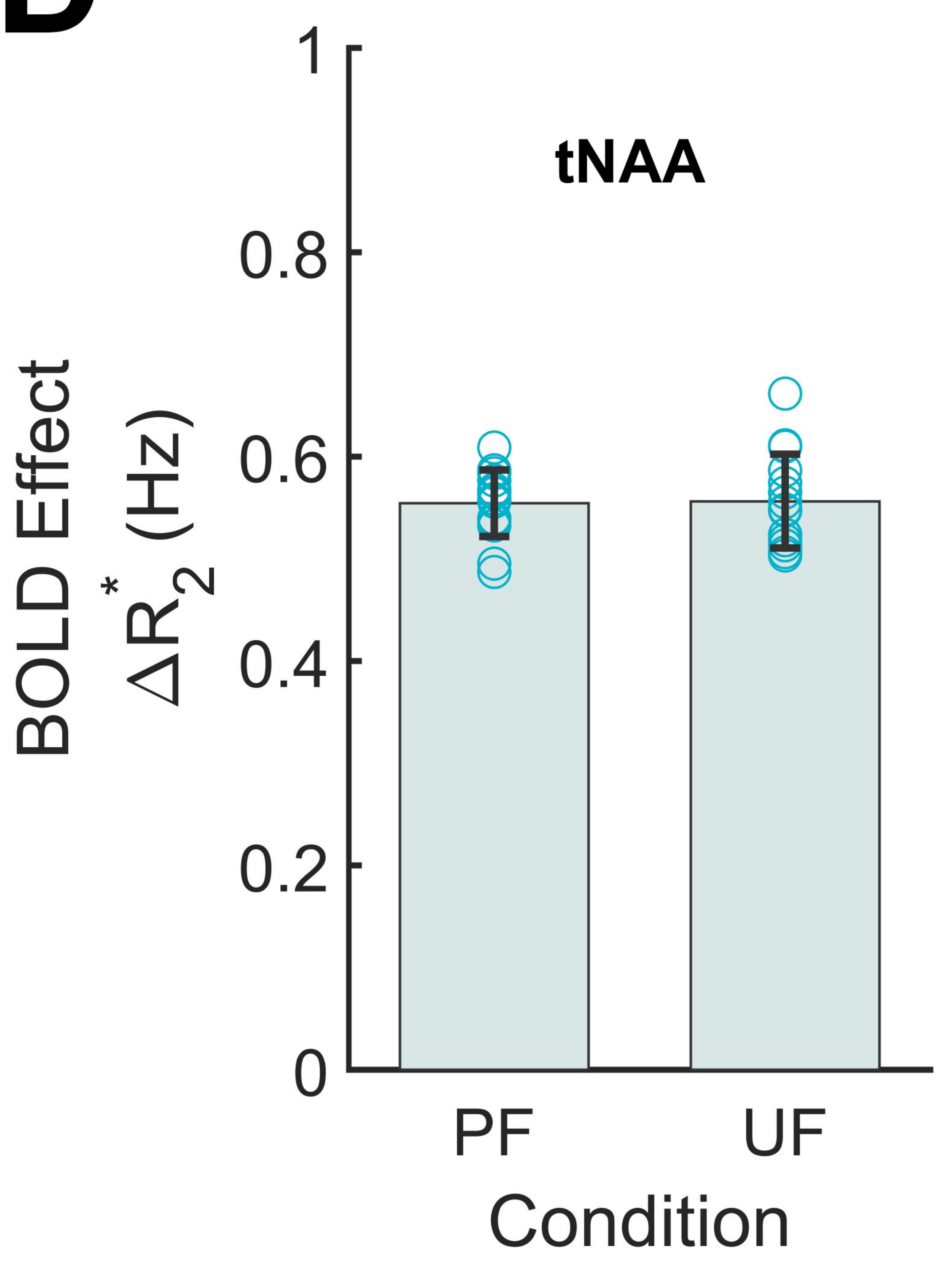

E
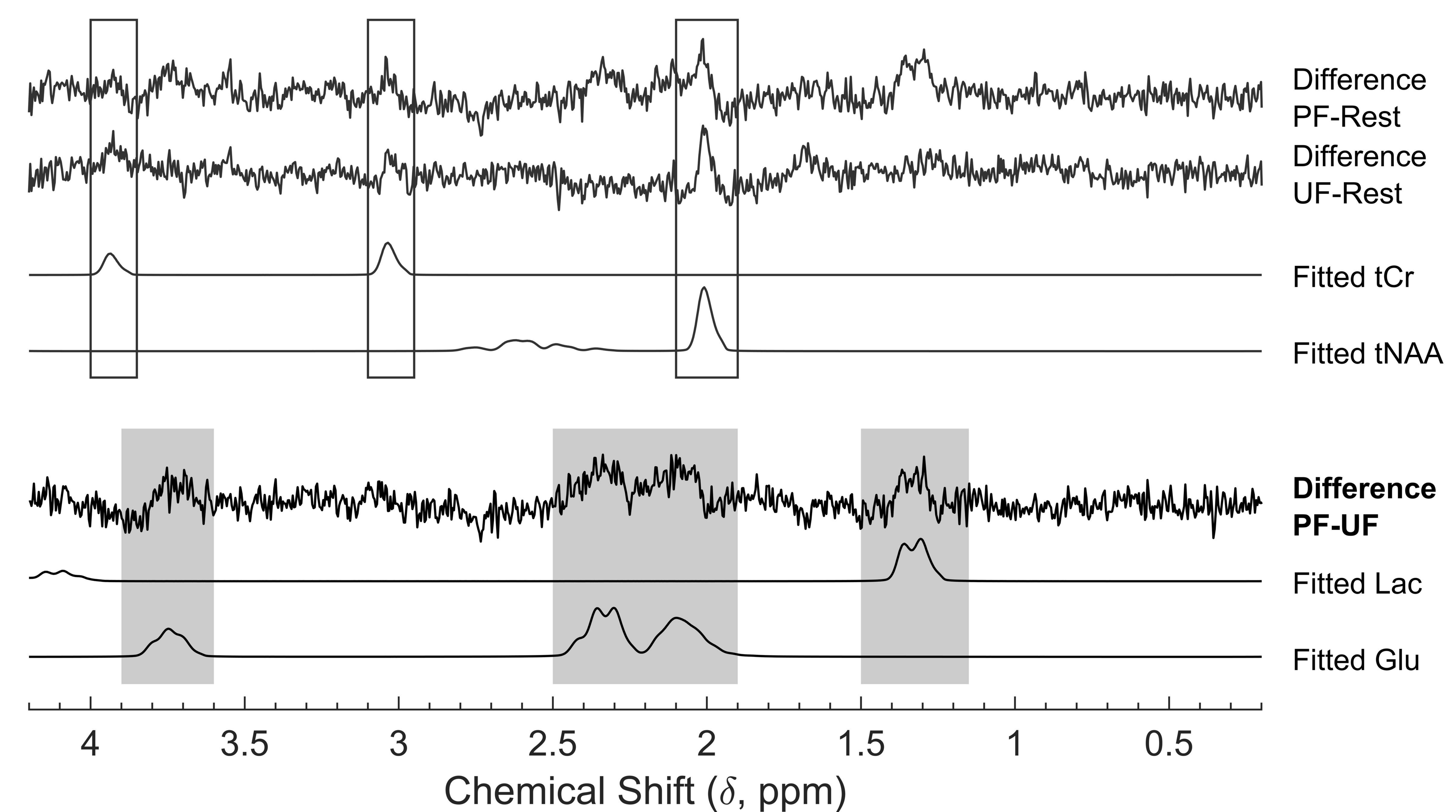



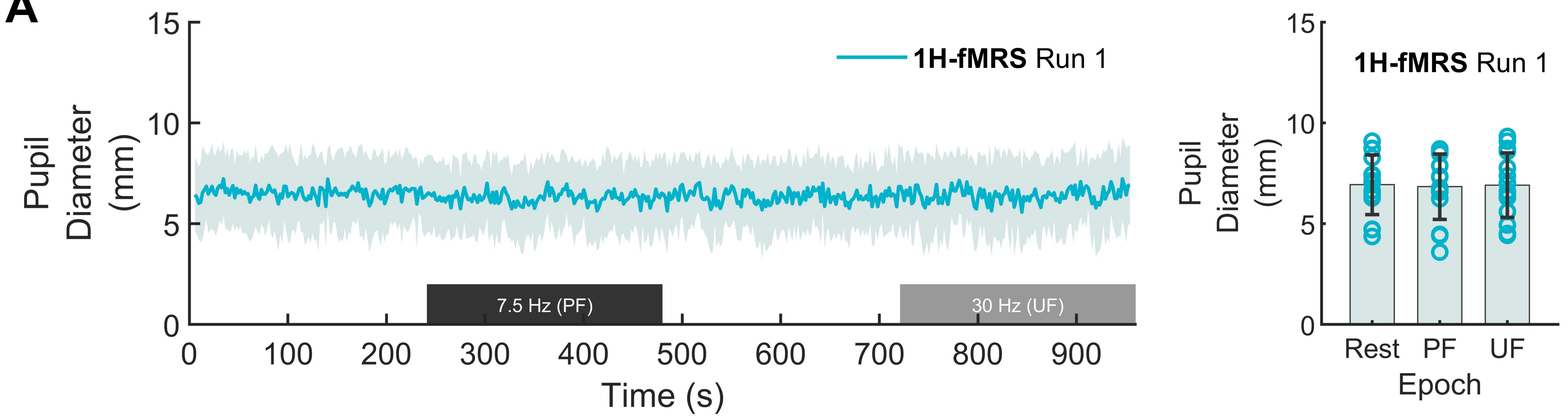

B

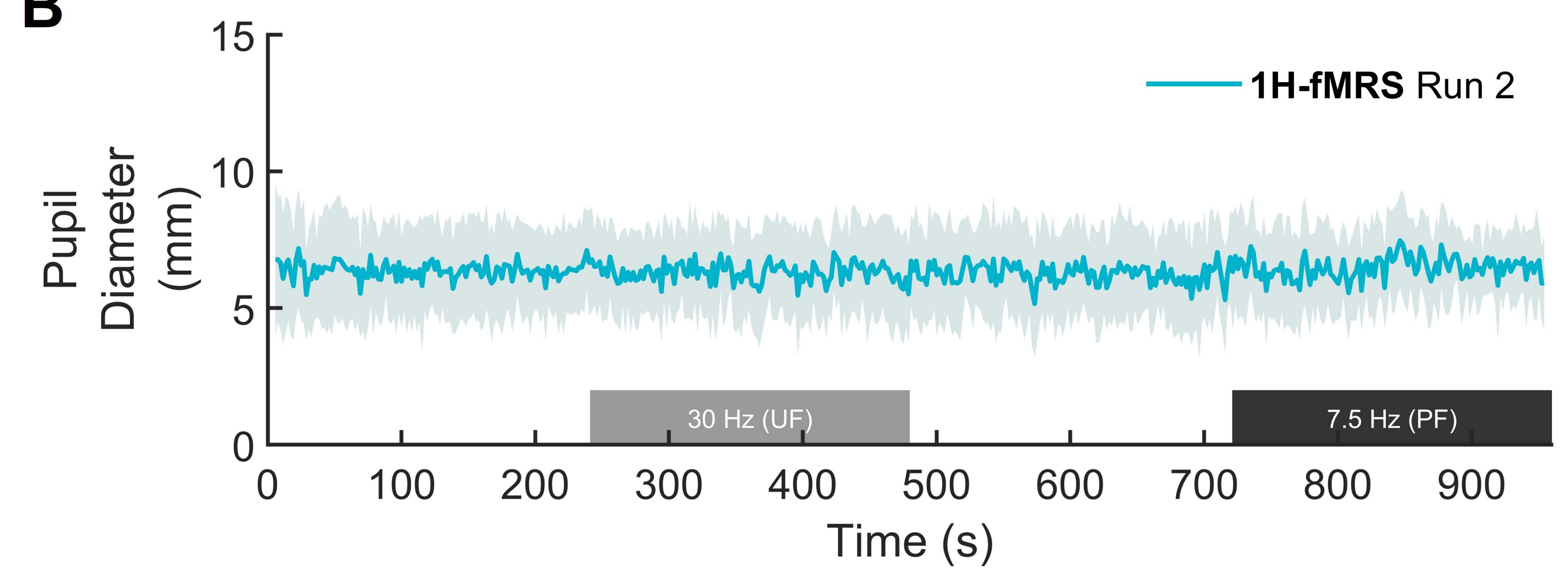

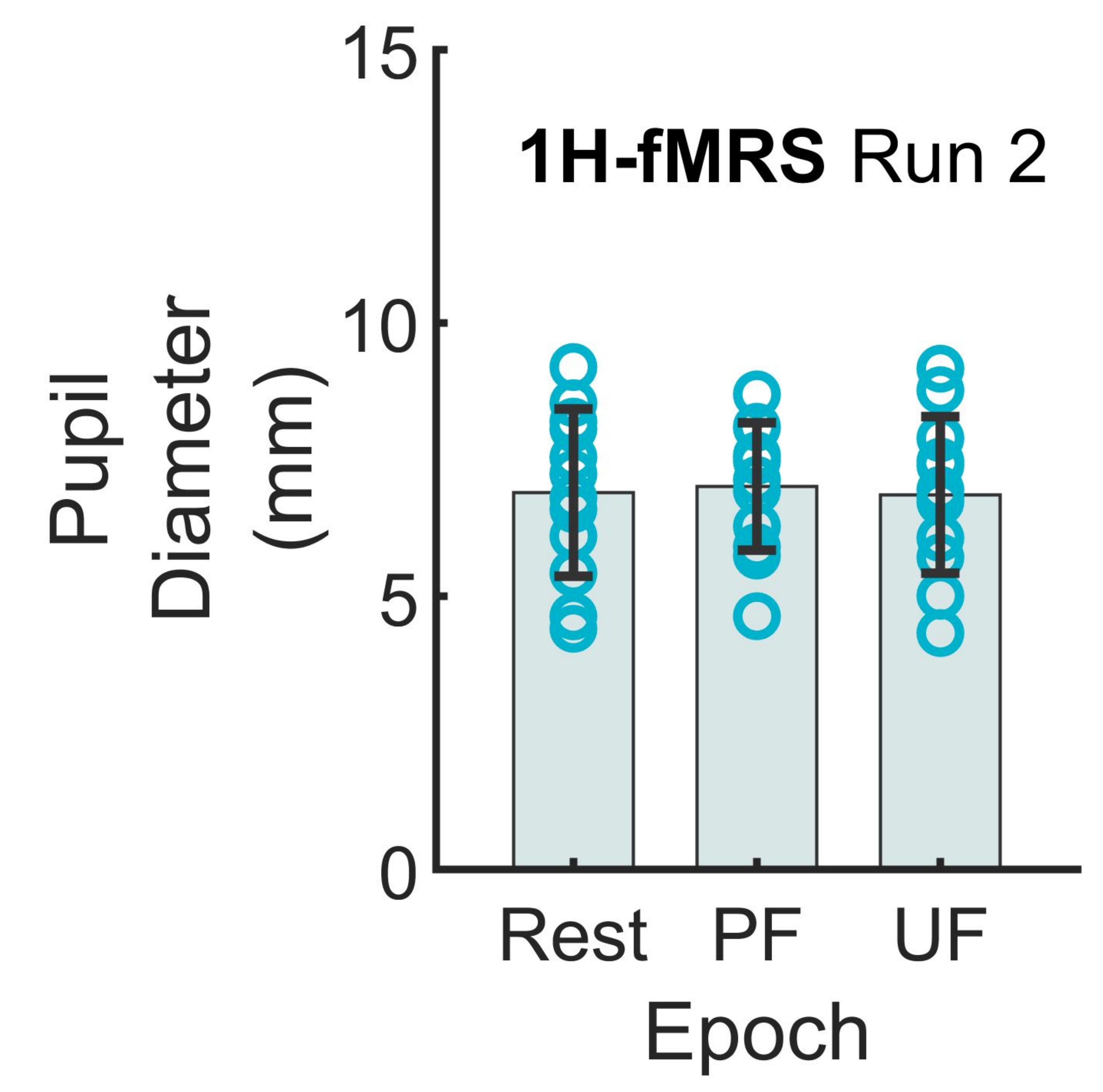




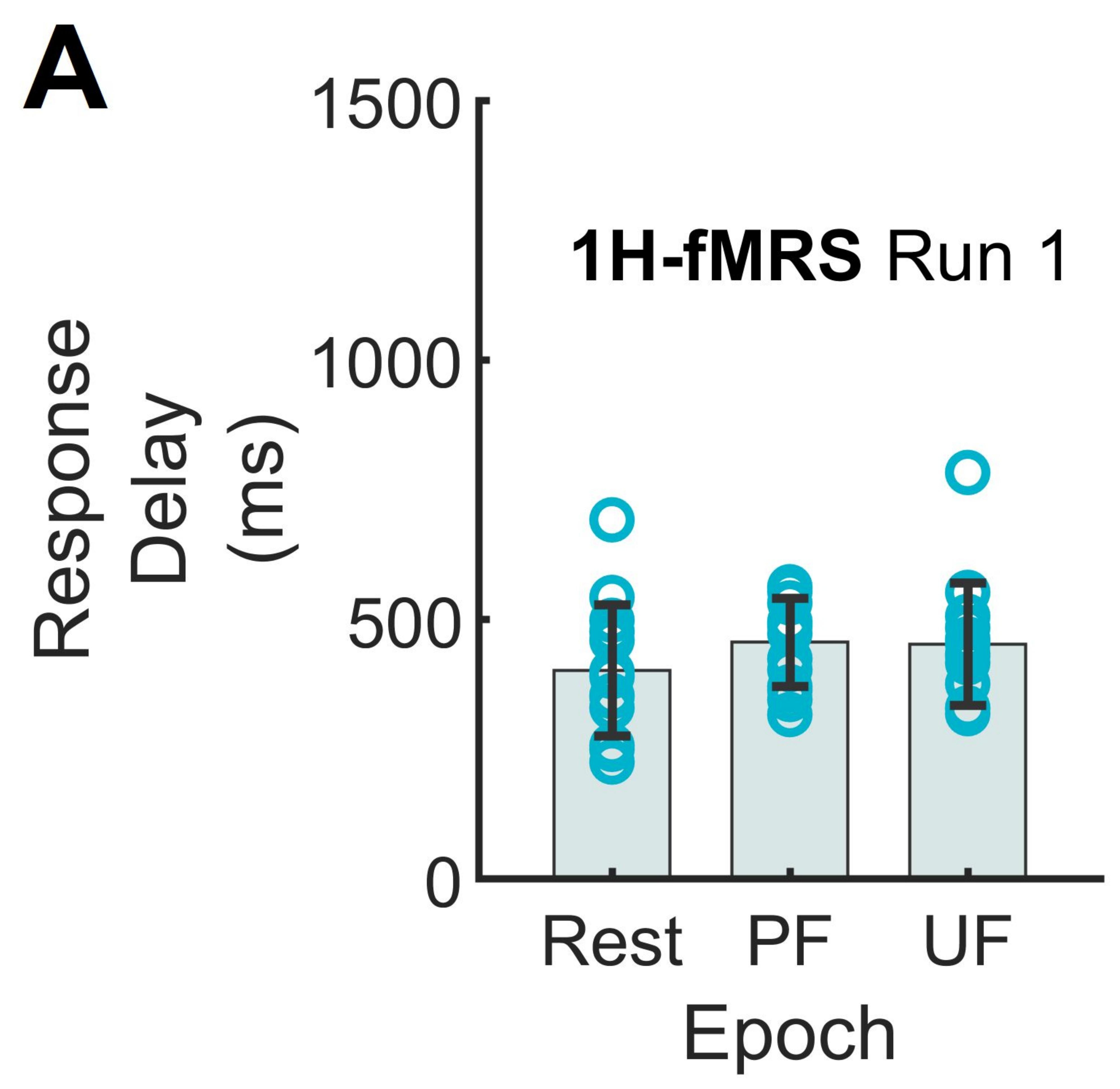

B
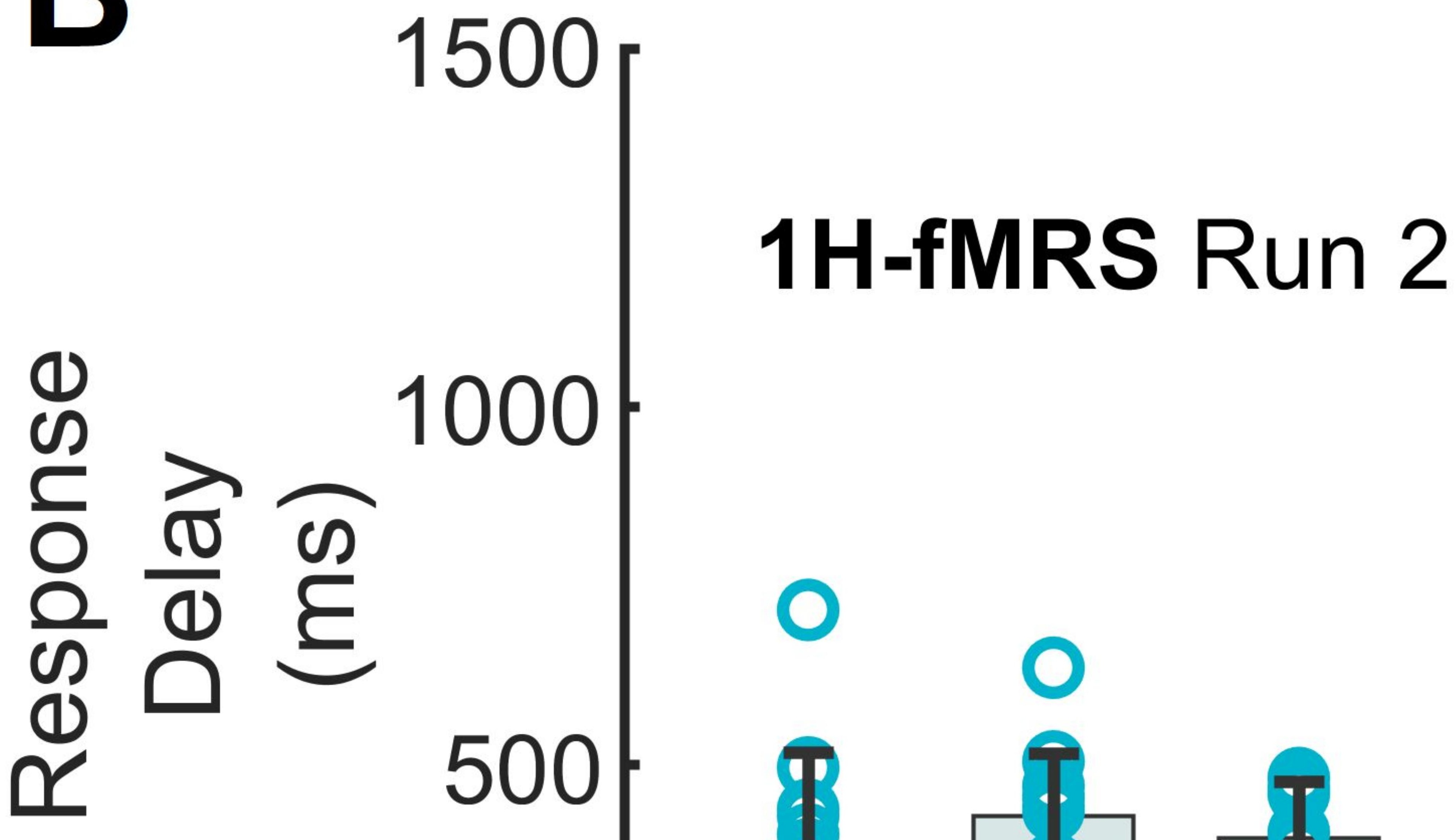

0

1000 .

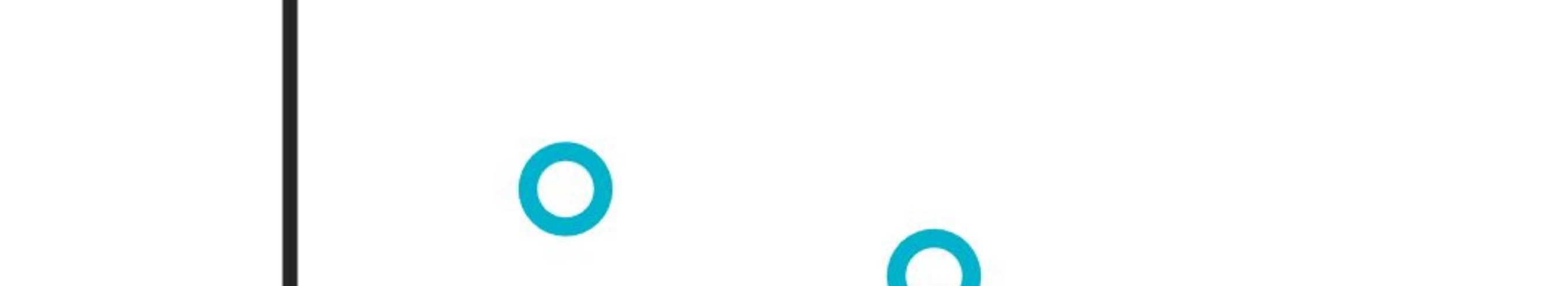

500

Rest PF UF

Epoch 
A

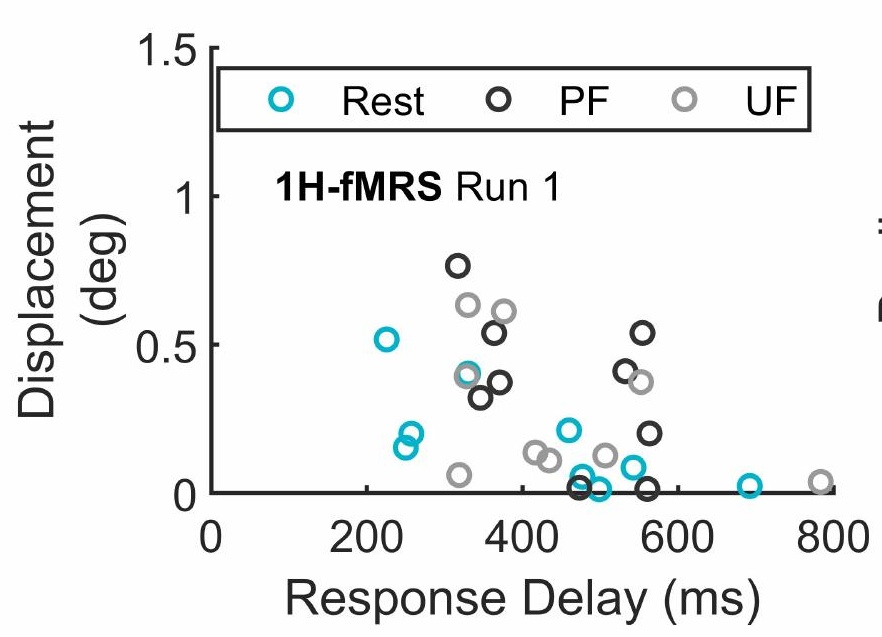

B
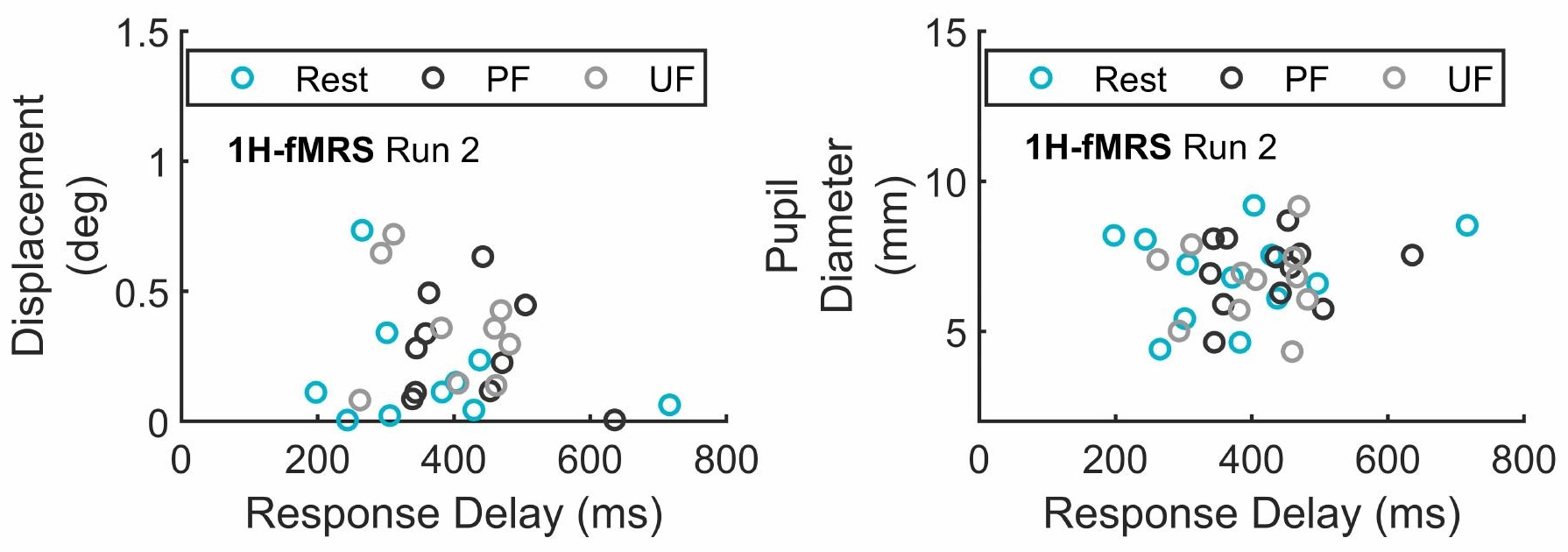
REST ( $N=32)$

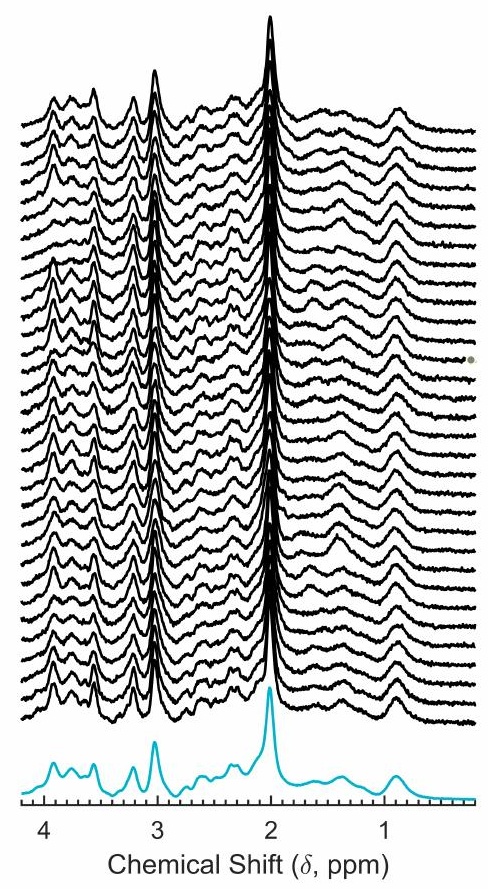

PF (N=16)

UF $(\mathrm{N}=16)$

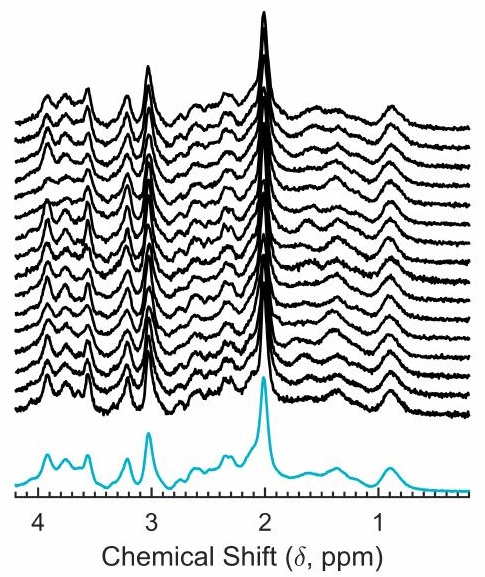

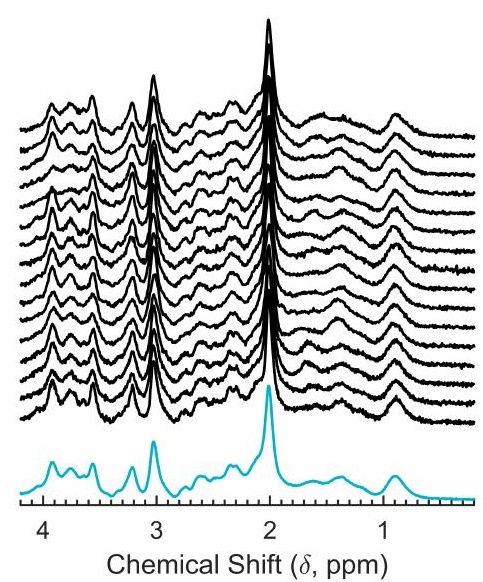

